Rhode Island College

Digital Commons @ RIC

$5-23-2020$

\title{
Dexmedetomidine Use in Alcohol Withdrawal Syndrome: A Retrospective Chart Review
}

Elizabeth Catherine Stefanilo

Follow this and additional works at: https://digitalcommons.ric.edu/etd

Part of the Nursing Commons

\section{Recommended Citation}

Stefanilo, Elizabeth Catherine, "Dexmedetomidine Use in Alcohol Withdrawal Syndrome: A Retrospective Chart Review" (2020). Master's Theses, Dissertations, Graduate Research and Major Papers Overview. 371.

https://digitalcommons.ric.edu/etd/371

This Major Paper is brought to you for free and open access by the Master's Theses, Dissertations, Graduate Research and Major Papers at Digital Commons @ RIC. It has been accepted for inclusion in Master's Theses, Dissertations, Graduate Research and Major Papers Overview by an authorized administrator of Digital Commons @ RIC. For more information, please contact digitalcommons@ric.edu. 
DEXMEDETOMIDINE USE IN

ALCOHOL WITHDRAWAL SYNDROME:

A RETROSPECTIVE CHART REVIEW

by

Elizabeth Catherine Stefanilo RN, BSN, CCRN

A Major Paper Submitted in Partial Fulfillment

of the Requirements for the Degree of

Master of Science in Nursing

in

The School of Nursing

Rhode Island College

2020 


\begin{abstract}
Research has shown that the abrupt termination of alcohol consumption by those described as having alcohol use disorder can place them at risk for alcohol withdrawal syndrome (AWS). The main goal of treating alcohol withdrawal syndrome is preventing the more severe symptoms such as delirium tremens, seizures, and death. Although, many other pharmacological treatments have been reviewed the use of benzodiazepines remains the standard of care. Dexmedetomidine (Precedex) has recently been added for the treatment of the hyperautonomic symptoms associated with alcohol withdrawal syndrome. While benzodiazepines reduce the occurrence of seizures in alcohol withdrawal patients, they have a vast number of negative side effects when administered in large quantities. Adding dexmedetomidine as an option for symptom management during delirium tremens may reduce the negative effect that copious quantities of benzodiazepines have on the individual. The purpose of this project then, was to determine if the use of Dexmedetomidine safely decreases the total dose of benzodiazepines administered to patients experiencing alcohol withdrawal. A retrospective chart review was conducted to explore the relationship between dexmedetomidine use and the total administered dose of benzodiazepines in patients admitted to a medical intensive care unit with severe alcohol withdrawal.
\end{abstract}




\section{Table of Contents}

Background/Statement of the Problem ................................................................. 1

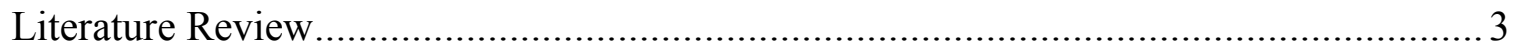

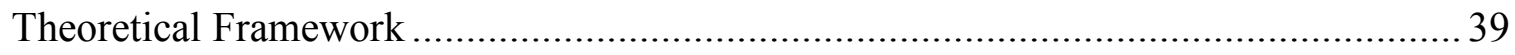

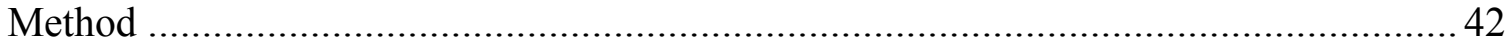

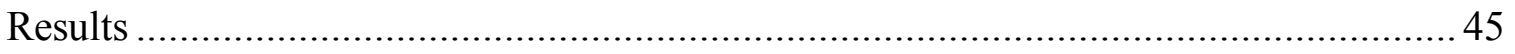

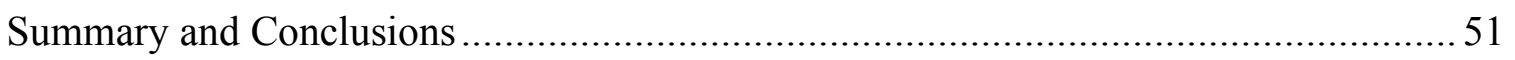

Recommendations and Implications for Advanced Nursing Practice ......................... 54

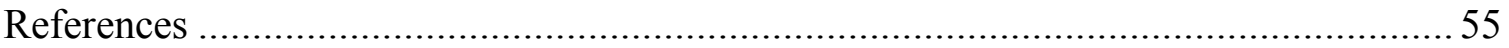


Dexmedetomidine Use in Alcohol Withdrawal Syndrome: A Retrospective Chart Review

\section{Background/Statement of the Problem}

Alcohol use disorder is a chronic relapsing brain disease characterized by an impaired ability to stop or control alcohol use despite adverse social, occupational, or health consequences (National Institute on Alcohol Abuse and Alcoholism, 2017). The abrupt termination of alcohol consumption by those described as having alcohol use disorder can place them at risk for alcohol withdrawal syndrome (AWS). To be classified as alcohol withdrawal a patient must meet the symptoms listed in the Diagnostic and Statistical Manual of Mental Disorders. These symptoms must result in clinically significant distress or impairment in normal functions (Schmidt et al., 2016). The literature suggests that alcohol withdrawal syndrome develops within 6-24 hours once the consumption of alcohol ceases or decreases. Alcohol withdrawal syndrome is a clinical condition characterized by symptoms of autonomic hyperactivity, such as, agitation, tremors, irritability, anxiety, hyperreflexia, confusion, hypertension, tachycardia, fever, and diaphoresis. (Mirijello et al., 2015). The withdrawal from alcohol is life threatening if not treated appropriately. If alcohol withdrawal persists to delirium tremens (DT's) there is an associated 5-10\% mortality rate (Guirguis et al., 2017). The main goal of treating alcohol withdrawal syndrome is preventing the more severe symptoms such as delirium tremens, seizures, and death (Mirijello et al., 2015). It is the "gold standard" in the management of AWS to use a symptom triggered approach when using pharmacological agents. Schmidt et al. (2016) concluded there is lack of available research to guide management of AWS. Although, many other pharmacological treatments have been reviewed the use of benzodiazepines remains the standard of care. Benzodiazepines reduce the incidences of seizures and delirium and shorten the duration 
of AWS. However, benzodiazepine therapy alone may not control alcohol withdrawal symptoms sufficiently and the increasing doses of such drugs is associated with excessive sedation, respiratory failure, worsening delirium, increased aspiration and intubation risks, increased length of stay and increased hospital costs.

Dexmedetomidine (Precedex) is an exceedingly selective $\alpha 2$-agonist that may diminish the hyperautonomic symptoms of alcohol withdrawal syndrome. It also provides light level of sedation as opposed to other agents used as treatment for AWS (VanderWeide et al., 2016).

There is increasing use of dexmedetomidine (Precedex) in the clinical setting for the management of alcohol withdrawal symptoms. The use of large doses of benzodiazepines for the control of withdrawal may result in over sedation, respiratory depression, and worsening delirium. dexmedetomidine, a sedative, has less effect on respiratory depression while still having optimal effects on anxiety, tachycardia and sedation; all of which are symptoms of withdrawal. Adding dexmedetomidine as an option for symptom management during delirium tremens may reduce the negative effect that copious quantities of benzodiazepines have on the individual. Therefore, the purpose of this project was to determine if the use of Dexmedetomidine impacts the dosage of benzodiazepines administered to patients admitted to the intensive care unit with severe alcohol withdrawal. 


\section{Literature Review}

A comprehensive literature review was completed using the search engines CINAHL and PubMed. The following key words where used for the search: alcohol use disorder, alcohol withdrawal syndrome, alcohol withdrawal assessment, alcohol withdrawal treatment, benzodiazepines, alcohol withdrawal and benzodiazepines, adverse effects of benzodiazepines, dexmedetomidine (Precedex), alcohol withdrawal and dexmedetomidine. Using these terms, many informational articles were presented and reviewed. Six articles were chosen based on the relevancy to this project. In this literature review, there will be four topics including: alcohol withdrawal syndrome, alcohol withdrawal assessment, treatment and management of alcohol withdrawal, and dexmedetomidine with alcohol withdrawal.

\section{Alcohol Use Disorder}

According to the National Survey on Drug Use and Health in 2015, 15.1 million adults ages 18 and older reported having alcohol use disorder, and of that 15.1 million, about 6.7 percent of adults who had AUD in the year of 2015 received treatment. An estimated 88,000 people die from alcohol-related causes annually, making alcohol the third leading preventable cause of death in the United States (National Institute on Alcohol Abuse and Alcoholism, 2017). Additionally, the definition of alcohol dependence has recently been amended. The American Psychiatric Association (APA) revised the Diagnostic and Statistical Manual of Mental Disorders (American Psychiatric Association, 2013) by combining both abuse and dependency disorders into a single distinct condition using the term alcohol use disorder (AUD). The DSM previously defined two distinct disorders, alcohol abuse and alcohol dependence. The updated definition states that alcohol use disorder is a chronic relapsing brain disease 
characterized by an impaired ability to stop or control alcohol use despite adverse social, occupational, or health consequences (National Institute on Alcohol Abuse and Alcoholism, 2017). Alcohol use disorder is now considered a single disorder with mild, moderate, and severe sub-classifications. The DSM-5 states that anyone meeting two of the eleven criteria they identified, is considered to have alcohol use disorder. Mild AUD is defined as the presence of 2-3 symptoms, moderate AUD is the presence of 4 to 5 symptoms, and severe is the presence of 6 or more symptoms (American Psychiatric Association, 2013).

Alcohol use disorder is classified from mild to severe based on the number of symptoms present. The presence of at least two of the following symptoms indicates alcohol use disorder: (1) Alcohol is often taken in larger amounts or over a longer period that was intended (2) There is a persistent desire or unsuccessful efforts to cut down or control alcohol use (3) A great deal of time is spent in activities necessary to obtain alcohol or recover from its effects (4) A strong desire or craving to use alcohol (5) Recurrent alcohol use resulting in failure to fulfill major role obligations at work or home (6) Continued alcohol use despite having persistent or recurrent social or interpersonal problems caused or exacerbated by the effects of alcohol (7) Important social, occupation, or recreational activities are given up (8) Recurrent alcohol use in situation in which it is physically hazardous, alcohol use is continued despite knowledge of having a persistent or recurrent physical or psychological problem that is likely to have been caused by alcohol (Sutton \& Jutel, 2016). 


\section{Alcohol Withdrawal Syndrome}

The abrupt termination of alcohol consumption by those described as having AUD can place them at risk for alcohol withdrawal syndrome (AWS). Alcohol withdrawal syndrome is a clinical condition characterized by symptoms of autonomic hyperactivity, such as agitation, tremors, irritability, anxiety, hyperreflexia, confusion, hypertension, tachycardia, fever, and diaphoresis. (Mirijello et al., 2015). Given alcohols short duration of action, withdrawal symptoms usually develop within 8 to 72 hours once the consumption of alcohol ceases or decreases (Schmidt et al., 2016).

Ethanol is a central nervous system depressant that causes behavioral excitation at low blood concentrations secondary to increased glutamate binding to N-methyl-Daspartate (NMDA) receptors (Jesse et al., 2016). At higher concentrations, ethanol causes acute intoxication by potentiation of the gamma-aminobutyric acid (GABA) especially in receptors with delta subunits. The delta subunits are distributed in the cerebellum, cortical areas, thalamic relay circuitry and brain stem which explains the intoxicating effects of alcohol (Jesse et al., 2016). Sutton and Jutel (2016) analyzed the neurobiology of alcohol withdrawal and point out that it is important to understand the chemical changes that occur when alcohol is introduced to the body to provide appropriate treatment for withdrawal. According to Sutton and Jutel (2016) both the neurotransmitter gamaaminobutyric acid (GABA) and the excitatory glutamate systems are affected by long term alcohol consumption. This may be due to the compensatory functional changes like the downregulation of GABA receptors and increased expression of NMDA receptors to maintain central nervous system homeostasis (Jesse et al., 2016). Abrupt cessation of alcohol will display the changes in receptors with a glutamate-mediated CNS excitation 
(Jesse et al., 2016). There is an unopposed neuronal excitation accounting for the automatic hyperactivity that occurs during AWS leading to neuropsychiatric complications such as delirium and seizures (Jesse et al., 2016). The upregulation of NMDA receptors and reduced GABA-A receptor inhibition explain most of the symptoms seen with withdrawal. The therapeutic approach to the treatment of alcohol withdrawal targets these receptor mechanisms (Jesse et al., 2016).

To be classified as having alcohol withdrawal a patient must meet symptom criteria listed in the Diagnostic and Statistical Manual of Mental Disorders. The DSM-5 suggests that two or more of the following symptoms must have developed within a few hours to five days after cessation of alcohol to be classified as alcohol withdrawal: diaphoresis, pulse rate greater than $100 \mathrm{bpm}$, increased hand tremor, insomnia, nausea, transient visual tactile or auditory hallucinations or illusions, psychomotor agitation, anxiety, or generalized tonic-clonic seizures. These symptoms must additionally result in clinically significant distress or impairment in normal functions (Schmidt et al., 2016). Severe alcohol withdrawal symptoms, including delirium tremens (DTs), may begin three to five days after cessation. (Guirguis, Richardson, Kuhn, \& Fahmy, 2017). There is wide variability in both onset and symptomology among patients experiencing alcohol withdrawal syndrome making it difficult for providers to predict the exact course of the syndrome.

Withdrawal from alcohol is life threatening if not treated appropriately. If alcohol withdrawal persists to delirium tremens (DT's) there is an associated $5-10 \%$ mortality rate (Guirguis et al., 2017). Untreated or undertreated delirium tremens will increase the mortality rate of patients up to $15 \%$ (Sutton \& Jutel, 2016). According to the DSM-5, a 
patient who meets the criteria for both alcohol withdrawal and delirium is considered to have delirium tremens. There is some incongruence in the literature of the true definition of delirium tremens. As early as the 1990's researchers defined AWS patients with delirium as having some level of confusion or disorientation, often accompanied by illusions, hallucinations, and agitation in addition to severe autonomic dysfunction (Schuckit, Tipp, Reich, Hesselbrock, \& Bucholz, 1995).

Challenges in Care of Alcohol Withdrawal Syndrome. Prolonged lengths of stay, increased risk for infectious complications, delirium, and the need for mechanical ventilation have all been reported as complications that may occur in patients experiencing alcohol withdrawal (Sutton \& Jutel, 2016). In a comprehensive literature review by Sutton and Jutel (2016), the authors note the challenges associated with the management of alcohol withdrawal syndrome, specifically those admitted to a critical care unit, were examined. Sutton and Jutel evaluated 107 peer reviewed articles, including one meta-analysis and 2 systematic reviews. The remaining 104 articles included observational studies, prediction tools and biomarkers, qualitative interviews, a protocol evaluation, a survey, reviews, and letters or commentaries (Sutton \& Jutel, 2016).

These authors concluded that there are many challenges in caring for patients with AWS, especially in identification of AWS (Sutton \& Jutel, 2016). Because clinical manifestations often resemble those of a critical illness, there is an alarming under recognition of patients in active withdrawal. Identifying critically ill patients who are in active alcohol withdrawal is challenging. Sepsis, intracranial hemorrhage, meningitis, stroke, traumatic brain injury, and metabolic derangements may all have signs and 
symptoms like that of alcohol withdrawal (Sutton \& Jutel, 2016). These conditions are also frequently seen in the intensive care unit. Comorbid patients taking medications such as beta-blockers, may mask the significant changes in vital signs that can occur with withdrawal. Differentiating between AWS and other disease states allows treatment for withdrawal to begin sooner. Sutton and Jutel (2016) further describe the implications that limited research regarding alcohol withdrawal in critical care patients has on current practice. Sutton and Jutel (2016) discuss the great need that exist for further research on every aspect related to alcohol withdrawal syndrome in critically ill patients. Specific recommendations for sedation-agitation or delirium detection scales for withdrawal in ICUs cannot be made with such limited research. A combination of the Confusion Assessment Method (CAM) for the ICU along with a sedation-sedation agitation scale may be beneficial in withdrawal patients admitted in critical care (Sutton \& Jutel, 2016).

Alcohol withdrawal can often lead to intensive care unit admissions due to its severity and complications. Currently, no universal practice guidelines exist to treat the critically ill patient experiencing alcohol withdrawal (Awissi, Lebrun, Coursin, Riker, \& Skrobik, 2013). In a recent systematic review, Awissi et al., (2013) examined alcohol withdrawal and delirium tremens in the critically ill. These investigators searched several databases for publications with high or moderate Grading of Recommendations Assessment, Development and Evaluation (GRADE) scores. Out of the 112 eligible articles, 34 were utilized based on inclusion criteria.

Results demonstrated that approximately $20 \%$ of admissions to the intensive care unit are contributed to excess alcohol consumption (Awissi et al., 2013). The authors also noted that identification of risk factors explicit for alcohol withdrawal could not be 
determined because no study prospectively evaluated all risk factors. Awissi et al. (2013) concluded that alcohol-dependent patients with a history of prior alcohol withdrawal are at highest risk for withdrawal symptoms. Several studies included in this review showed a high rate of Intensive Care Unit (ICU) admission attributable to alcohol withdrawal, but once admitted their length of stay was no higher than medical or trauma patients. Several studies also assessed financial estimates related to alcohol withdrawal. Costs were significantly higher in alcohol-related ICU admissions compared to non-alcoholics (\$52,527 vs \$43,156). Awissi et al. (2013) found that ICU patients experiencing withdrawal having a longer duration of mechanical ventilation, more frequent pneumonia, urinary tract infections, sepsis and septic shock. Notably, Awissi, et al. (2013) point out that although these complications may be associated with alcohol use, immune downregulation is also associated with benzodiazepine use.

Overall, the investigators determined that data related to long term outcomes related to this patient population is limited. The studies included in this review further demonstrated evidence that patients admitted to the ICU with withdrawal, were often seen again in the emergency room within two years (Awissi et al., 2013). Awissi et al., (2013) concluded that research in this area needs to be continued. Approximately $20-40 \%$ of hospitalized patients and 50-60\% of trauma patients are affected by chronic alcoholism. Despite the prevalence of alcohol withdrawal within a hospital, there is little high-quality data to guide treatment. Further research should be conducted on how to best prevent, diagnose, and treat alcohol withdrawal in the critically ill patient (Awissi et al., 2013). 


\section{Early Identification of Alcohol Withdrawal Syndrome}

A majority of literature that examines alcohol withdrawal has concluded that early identification of daily alcohol consumption in patients who were acutely hospitalized is important to assess the possible severity of their withdrawal. This is imperative if providers are to effectively manage alcohol withdrawal (Sutton \& Jutel, 2016).

Foy, Kay and Taylor (1997) studied patients with episodes of alcohol withdrawal, who were admitted to Newcastle Mater Misericordia Hospital on a general medicine unit. These authors conducted an observational study of alcohol withdrawal in patients admitted for numerous medical conditions, as well as those admitted specifically for alcohol withdrawal. The purpose of their study was to examine these patients to determine the natural history of their withdrawal. Foy et al. (1997) aimed to evaluate the incidence of seizures, hallucinations and delirium, along with the risk factors for such events. They monitored patients' withdrawal based on the following: timing and severity of noteworthy events; patients who were most at risk for more serious event; what to expect when a serious event occurred; and the ability to assess patient's risk factors to predict what outcomes were most likely.

The researchers noted that out of 539 participants, alcohol withdrawal was not associated with alcohol mortality but did extend the length of stay by a median of 4 days (Foy et al., 1997). Foy et al. (1997) concluded that the principal element in alcohol withdrawal management is the early detection and management of patients who have the potential for withdrawal. Early initiation of increased monitoring was associated a significant reduction in risk of complications. Patients whose monitoring was delayed had 
three times the number of complications compared to those whose treatment was not delayed (Foy et al., 1997).

Identifying patients at risk for alcohol withdrawal is key to effective and early treatment of withdrawal in the hospital (Sutton \& Jutel, 2016). A patient's history of alcohol consumption is difficult to obtained and not always detailed enough. Several screening tools have been developed to screen for alcohol use disorder including the Alcohol Use Disorder Identification Test (AUDIT), the Michigan Alcoholism Screening Test, and the CAGE questionnaire. Although these tools exist, they have never been tested in critically ill patients. Lack of consistency has been reported with creating a reliable diagnostic criteria tool for withdrawal. Development of a tool that critical care nurses could use to identify patients with AUD, could prevent their mild withdrawal from progressing to severe (Sutton \& Jutel, 2016). Past medical history has proven to be the strongest predictor for progression to alcohol withdrawal (Schmidt et al., 2016).

Treatment of alcohol withdrawal could be optimized if patients at a higher risk could be identified. Prediction and prevention become cornerstones in avoiding severe withdrawal or delirium tremens. Eyer et al. (2011) aimed to develop a prediction model for withdrawal seizures and delirium tremens during moderate to severe alcohol withdrawal syndrome. This study was a secondary analysis of a cohort of adult patients admitted to a toxicological unit for alcohol withdrawal. A total of 827 patients received a score-guided pharmacological treatment with oral clomethiazole to achieve minimal withdrawal symptoms. Patient's withdrawal severity was assessed using a validated 11item withdrawal score. 
Data was collected on demographics, withdrawal history, alcohol or drug use, laboratory data on admission, presence of cirrhosis, blood pressure and heart rate, and presence of any medical or psychiatric comorbidities. The amount of daily alcohol consumption was reported by the patient and calculated (grams ethanol $=$ volume of drink [ml] x $0.8 \times$ alcohol content [\%]/100). Complications, length and quantity of medical treatment, and AWS scores upon and during treatment were also collected. Eyer et al. (2011) compared the patient's withdrawal history, clinical and some analytical parameters of patients who developed delirium tremens and/or withdrawal seizures, and those who did not (Eyer et al., 2011).

Of the 827 charts reviewed, 46 patients (5.6\%) developed delirium tremens and 61 patients (7.4\%) developed withdrawal seizures. First the researchers compared patients who underwent a withdrawal seizure with those who did not. There was a higher incidence of seizures in patients with previous withdrawal episodes (59 vs $41 \%$; $p=$ 0.007). Patients who were positive for seizures also had more frequent episodes of delirium tremens ( 15 vs $5 \% ; p=0.004)$. On admission, patients who underwent seizures had significantly lower serum potassium ( $4.0 \pm 0.4 \mathrm{vs} 4.2 \pm 0.5 \mathrm{mmol} / \mathrm{L} ; p=0.029)$, lower platelets $(150 \pm 81 \mathrm{vs} 189 \pm 94 \mathrm{~g} / 1 ; p=0.001)$, a later climax of withdrawal symptoms (34 \pm 36 vs $18 \pm 19 \mathrm{~h} ; p<0.001)$, and required longer medical treatment (127 \pm 87 vs $86 \pm 49 \mathrm{~h} ; p$ $<0.001$ ) (Eyer et al., 2011).

In comparing patients who experienced delirium tremens and those who did not, there were significant differences found. Patients who underwent delirium tremens were a higher age (49 \pm 10 vs $45 \pm 10 \mathrm{a} ; p=0.023)$, had lower approximated daily ethanol intake (164 \pm 139 vs. $236 \pm 169 \mathrm{~g} ; p=0.007)$, and had a higher frequency of related seizures (20 vs. $7 \% ; p=0.004)$. Delirium tremens patients were admitted less frequently electively for 
alcohol withdrawal (11 vs. $32 \% ; p=0.002)$ but were admitted more significantly more with withdrawal seizure being the cause (46 vs. $14 \%$; $p<0.001$ ). Patients with delirium tremens showed significantly lower serum sodium $(138 \pm 6$ vs. $140 \pm 5 \mathrm{mmol} / \mathrm{l} ; p=0.023)$ and potassium $(3.8 \pm 05$ vs. $4.2 \pm 0.5 \mathrm{mmol} / 1 ; p<0.001)$, lower platelet count (119 $\pm 62 \mathrm{vs}$. $190 \pm 93 \mathrm{~g} / \mathrm{l} ; p<0.001)$, and had a lower serum ethanol concentration (1.4 $\pm 1.7 \mathrm{vs}$. $2.4 \pm 1.6 \mathrm{~g} / 1 ; p<0.001)$ (Eyer et al., 2011).

In the multivariable regression model, significant predictors for seizures during withdrawal were delayed climax of withdrawal severity (OR for every increase of 10h: 1.23; 95\% CI: 1.1-1.4; $p<0.001)$ and seizures as cause of admittance (OR 2.6; 95\% CI: 1.4-4.8; $p$ $=0.002)$. The c-index of this prediction model was 0.73 (95\% CI: 0.66-0.88). Significant predictors for occurrence of delirium tremens at admission were lower serum potassium (OR per an increase of $1 \mathrm{mmol} / 1: 0.33 ; 95 \% \mathrm{CI}: 0.17-0.65 ; p=000.1)$ and a lower platelet count (OR per an increase of 100.000: 0.42; CI 0.26-0.69; $p=0.001$ ). The c-index of this prediction model was 0.81 (95\% CI: 074-0.87) (Eyer et al., 2011).

This study displays the importance of obtaining a thorough addiction-related history. Multivariable logistic regression model revealed multiple factors that could predict delirium tremens or withdrawal seizures. The study by Eyer et al. (2011) provides an important starting point for early identification of alcohol withdrawal complications (Eyer et al., 2011).

Currently there are no pathognomonic clinical signs or symptoms of alcoholism. One way to identify alcohol use disorder is by using the previously mentioned screening tools. The use of alcohol abuse biomarker may be an accurate and objective way to diagnosis alcohol use disorder (Jastrzebska et al., 2016). Two kind of alcohol biomarkers exist. State markers are measures that allow evaluation of a patient's history of alcohol 
consumption and trait markers reveal a person's inherited risk of developing alcoholism due to chronic exposure (Jastrzebska et al., 2016). Researched biomarkers linked with alcohol use disorder include: $\gamma$-glutamyl transferase (GGT), aspartate aminotransferase (AST), alanine aminotransferase (ALT), mean cell volume (MCV), and carbohydratedeficient transferrin (CDT) (Jastrzebska et al., 2016). More recent research has revealed potential biomarkers with a more accurate reflection of excessive alcohol intake. Ethyl glucuronide (EtG) and ethyl sulfate (EtS) are direct conjugated metabolites of ethanol, if present, can indicate recent alcohol consumption (Jastrzebska et al., 2016). Following alcohol intake, acetaldehyde (the first product of oxidative metabolism of alcohol), accumulates in the red blood cells. The whole blood-associated acetaldehyde assay (WBAA) has potential to test for heavy alcohol consumption (Jastrzebska et al., 2016). Phsophatidylethanol (PEth) represents an abnormal cellular membrane phospholipid formed only in the presence of alcohol and serves as an alcohol consumption biomarker (Jastrzebska et al., 2016). Further research on biomarkers related to alcohol consumption could assist in the treatment of alcohol use disorder.

Not every patient that has alcohol use disorder will progress to alcohol withdrawal, just as not every patient who experiences alcohol withdrawal will undergo delirium tremens. The ability to predict the patients that will progress to severe alcohol withdrawal is imperative in providing early interventions. Researchers have taken some of the previously mentioned prediction factors and created tools used for identifying patients at risk of withdrawal. Although these tools have been validated in medical patients, no single tool has proved reliable in critical care patients (Sutton \& Jutel, 2016). 


\section{Assessing Alcohol Withdrawal Syndrome}

Once alcohol withdrawal syndrome begins, early and frequent assessment of symptoms is crucial to avoid complications. Several withdrawal or agitation scales having been identified that serve as a guide to objectively rate symptom severity or aid treatment in patients experiencing alcohol withdrawal. Most frequently used is the Clinical Institute Withdrawal Assessment (CIWA-Ar), Riker Sedation Agitation Scale (SAS), Minnesota Detoxification Scales (MINDS), Richmond Sedation Agitation Scale (RASS), and the Confusion Assessment Scale (CAM).

One scale utilized in assessing AWS, specifically in the intensive care unit, is the Richmond Agitation-Sedation Scale (RASS). The RASS is a 10-point scale with four levels of anxiety or agitation $(+1$ to +5$)$, one level to denote a calm and alert state $(0)$, and 5 levels of sedation (-1 to -5) (Sessler et al., 2002). It was designed by the collaborative efforts of critical care doctors, nurses, and pharmacists. Sessler et al. (2002) investigated the interrater reliability and validity of the RASS on adult patients admitted to intensive care units. Patients in the medical respiratory ICU, neuroscience ICU, coronary ICU, surgical trauma ICU, and cardiac surgery ICU were evaluated. Patients were excluded if a neuromuscular blockade or paraplegia was present, they were on airborne precautions, or were visually or hearing impaired.

During phase one of this study five investigators assessed the patients following the steps included on the RASS. All investigators independently recorded RASS scores at six separate occasions during May to September 1999. During phase two, inter-rater reliability was evaluated after implementation of RASS into a medical ICU in December 1999. A total of 192 patient encounters in 172 patients were evaluated. Scores ranged 
from -5 to +3 , with almost $43 \%$ of those scores being in the sedation range. Results of phase one showed that RASS scores were lower for mechanically ventilated patients ( $p<$ $0.0001)$ and for patients receiving continuous sedative medications $(p<0.001)$. RASS scores varied among the different critical care units $(p<0.001)$. Excellent inter-rater reliability was demonstrated for RASS scores among all critical care units $(\mathrm{ICC}=0.956$ $[0.948])(\varkappa=0.73[0.71,0.75])$. Inter-rater reliability was high for all pair-wise comparisons between investigators $(r=0.944-0.973)(\varkappa=0.65-0.80)$. As a validity measures, the mean RASS score recorded for four investigators was correlated highly $(\mathrm{r}=$ 0.93, $p<0.0001)$ Strong correlation between investigator RASS and visual analog scale score confirmed validity of RASS for all critical care groups $(\mathrm{r}=0.84-0.98, p<0.0001)$. In phase two, validity of RASS after implementation was demonstrated by a strong correlation between RAS and the Sedation Agitation Scale (SAS) $(\mathrm{r}=0.78, p=<0.0001)$, Ramsay sedation scale score $(\mathrm{r}=-0.78, p<0.001)$, and Glasgow Coma Scale scores $(\mathrm{r}=$ $0.79, p<0.0001$ ) (Sessler et al., 2002) demonstrated great inter-rater reliability and validity across a variety of adult ICU patients in hopes to enhance acceptance of the RASS by physicians, nurses, and pharmacists (Sessler et al., 2002).

The Riker Sedation-Agitation Scale (SAS) has been utilized in patients experiencing alcohol withdrawal in intensive care units. Scores range from zero (unarousable) to seven (dangerous agitation). One study examined the use of SAS scores on patients admitted to a medical ICU for the treatment of alcohol withdrawal. Gold, Rimal, Nolan, and Nelson (2007) assessed patients admitted to Bellevue Hospital medical ICU with SAS scores of 5 or greater that were managed with benzodiazepines. With no protocol in place to manage withdrawal, the SAS and symptom-triggered benzodiazepine 
dosing was used to create guidelines. Researchers aimed to detail the characteristics and outcomes of alcohol withdrawal patients before guideline directed therapy and after. Following implementation of the AWS protocol there was an increase in total benzodiazepine dose within the first 24 hours of withdrawal. There was also an increased use of phenobarbital $(58 \%$ vs. $17 \% p<.001)$. There was a significant reduction in the use of mechanical ventilation post-guideline $(21.9 \%$ vs. $47.3 \% ; p=.008)$. The two subjects that did require intubation post-guideline, were not managed according to guidelines. There was also a correlation between ICU length of stay and total amount of benzodiazepines administered post-guidelines ( $\mathrm{r}=.48 ; p=.008)$ (Gold et al., 2007).

Another method of evaluating the course of alcohol withdrawal is the Minnesota Detoxification Scales (MINDS) developed by DeCarolis, Rice, Ho, Willenbring, and Cassaro (2007). As stated previously, the CIWA-Ar protocol is only ideal for patients with the ability to communicate with the provider (DeCarolis et al., 2007). The MINDS score was created to reflect treatment of severe alcohol withdrawal in patients admitted to intensive care units. In comparison to the CIWA protocol, which is lengthy and takes approximately 5-15 minutes to complete, the MINDS score was determined to take 3-5 minutes to complete (DeCarolis et al., 2007). In a study by DeCarolis et al. (2007) the MINDS score was utilized to compare outcomes in patients admitted to the ICU with alcohol withdrawal. Thirty-six patients with 40 ICU admissions for AWD were examined for symptom control, total dose of benzodiazepines, amount of time receiving continuous benzodiazepine infusion, length of ICU and hospital stay, polypharmacy, and complications of treatment. Data was collected both before and after the implementation of a symptom-driven benzodiazepine protocol, MINDS. It was concluded that the 
MINDS protocol was associated with decreased time required to control severe symptoms and benzodiazepine dose needed (DeCarolis et al., 2007). Due to the ambiguity of assessing and consequently treating patients in the pre-protocol group, both delayed symptom control and higher doses of benzodiazepines were seen. The development of the MINDS protocol for severe AWD has both improved care and reduced risks associated with alcohol withdrawal syndrome (DeCarolis et al., 2007).

The Clinical Institute Withdrawal Assessment (CIWA-Ar) uses the DSM-5 categories and assigns them numerical scores to measure the severity of alcohol withdrawal. The CIWA scale is the most commonly used scale despite the limited research that has been conducted to validate it in the critically ill population (Sutton \& Jutel, 2016). The CIWA scale is a 10-item assessment tool measuring tremor, sweating, anxiety, and other signs of withdrawal. Scores range from 0 to 67 ; scores lower than 8 indicate mild withdrawal and rarely require medication. Moderate withdrawal is defined as a CIWA score of 8-15 and these patients tend to respond to modest doses of benzodiazepines. Lastly, a CIWA score of greater than 15 reflects severe alcohol withdrawal and require intense monitoring to avoid complications (Schuckit, 2014).

The CIWA scale can be utilized on patients prior to the progress to delirium tremens, multiple drug therapy, and possible intubation. Once a patient reaches that level of severity in withdrawal the CIWA scale proves no longer useful. Based on their review, Sutton and Jutel (2016) recommend the use of the Confusion Assessment Method scale in conjunction with a sedation-agitation scale to assess patients who are receiving multi-drug therapy or mechanically ventilated. 
Littlefield et al. (2018) conducted a study that evaluated the correlation between the CIWA-Ar scale the MINDS. In 2011, Yale New Haven Hospital implemented the Yale New Haven Alcohol Withdrawal Protocol (YAWP) to assess patients in alcohol withdrawal admitted to intensive care units. The YAWP pairs a modified Minnesota Detoxification Scale (mMINDS) with 3 benzodiazepine-based treatment strategies. The modifications made to the MINDS included detailed definitions when assessing tremor, delusions, and hallucinations using the Richmond Agitation Sedation Scale. In a singlecenter prospective correlation study performed at a large academic center, researchers collected information from 30 patients admitted to Yale New Haven Intensive Care Unit who were assessed daily with both the CIWA-Ar scale and mMINDS. The CIWA-Ar tool has only been validated in uncomplicated alcohol withdrawal, while the MINDS paired with a symptom-triggered benzodiazepine regimen has been researched for utilization in severe alcohol withdrawal. As stated previously, the MINDS scoring tool was developed to provide a more consistent assessment tool especially for those requiring ICU admission.

Littlefield et al. collected a total of 185 CIWA and mMINDS scores. The primary end point was evaluated by using the Pearson correlation coefficient. Correlation was defined as strong if the Pearson correlation coefficient was greater than 0.80 , moderate if between 0.50 and 0.80 , and weak if less than 0.50 . The Pearson correlation coefficient across all scores was found to be 0.82 , indicating a strong correlation, especially with values of 10 or less. Correlations were also evident for tremors (0.98), agitation (0.84), and orientation (0.87). Weak correlations were for tactile (0.07), auditory $(-0.07)$ and visual (0.04) hallucinations. 
The CIWA scoring tool uses a score of 0-7 whereas the mMINDS uses a score of 0-4 for each parameter. The variance in the rating scale for each parameter, there may be inconsistencies across scorers, leading to low interrater reliability in clinical practice. In 2007, DeCarolis and colleagues developed the MINDS scoring tool to provide more objective measures when assessing AWS in ICU level patients. The MINDS tool has not been studied in patients with less than severe alcohol withdrawal. At Yale New Haven Hospital, the CIWA scale is used in general inpatient units and the mMINDS is used in the step-down and ICU units. With the use of two distinct scoring tools, transition of care for patients in AWS is difficult. Littlefield et al. showed a strong relationship between the two scoring tools especially in patients with CIWA scores less than 10. Unfortunately, as the severity of AWS worsened, the correlation decreased. Variability found in the scores is related to the patient's ability to answer and respond to commands. As the AWS increases in severity, the patient becomes less likely to participate in the assessment. This may lead to a variability in the scoring tools at higher values. Hopefully, this study will improve the transition of care of AWS patients between units.

Recent research has shown that a symptom driven protocol can be effective. Utilization of a CIWA-Ar scale or an alternative protocol is likely to improve outcomes for patients with alcohol withdrawal (Mirijello et al., 2015). Regardless of the manner, the severity of symptoms associated with alcohol withdrawal must be closely monitored to identify the most effective treatment and prevent over sedation or other complications (Schuckit, 2014). 


\section{Treatment and Management of Alcohol Withdrawal Syndrome}

Once a patient has been admitted to the hospital for the treatment of AWS, symptom assessment and pharmacologic therapy must begin. According to (Myrick, 1998) inpatient detoxification from alcohol is the safest and most consistent treatment. The main goal of treating alcohol withdrawal syndrome is to control agitation all while preventing the more severe symptoms such as delirium tremens, seizures, and death (Mirijello et al., 2015). Supportive care should also be provided by increasing motivation in patients to maintain sobriety and to enroll them into a relapse prevention program.

Both non-pharmacological and pharmacological approaches benefit the individual in acute withdrawal. Some non-pharmacological interventions according to Mirijello et al., (2015) include reorientation to surroundings, decreasing stimulation, and emotional guidance. Routine examination of the patient in alcohol withdrawal should include blood alcohol concentrations, complete blood count, renal function tests, electrolytes, glucose, liver enzymes, urinalysis, and urine toxicology (Mirijello et al., 2015).

General treatment includes correcting hydration status, blood glucose checks, and replacement of electrolytes. Metabolic abnormalities are common in patients in acute alcohol withdrawal. Electrolyte disturbances should be corrected immediately, along with vitamin supplementation, as a causal relationship between hypomagnesia and the occurrences of seizures or delirium has been shown (Myrick, 1998). Other vitamin and electrolyte disturbances have been noted to occur during alcohol withdrawal. Thiamine levels are frequently deficient which can lead to Wernicke's encephalopathy (Schmidt et al., 2016). Wernicke's encephalopathy displays as an alerted mental status, ophthalmoplegia, and ataxia. Treatment includes high doses of thiamine intravenously 
(Schmidt et al., 2016). Hyperhomocysteinemia is thought to be caused by chronic alcohol use and can lead to folate deficiency. Folate supplementation is recommended through the administration of a multivitamin (Schmidt et al., 2016). Inadequate nutrition and poor hydration status are common complications of chronic alcohol use. Hypokalemia, hypophosphatemia, and hypomagnesemia can all be seen in alcohol withdrawal, repletion of all is recommended (Schmidt et al., 2016).

The "gold standard" of AWS management is to use a symptom triggered approach when using any pharmacological agent (DeCarolis et al., 2007). Combining the assessment scales noted with symptom-triggered benzodiazepine dosing can provide personal adaptation of medication dosing in AWS.

A treatment plan that is based on both an objective withdrawal severity scale and a subjective assessment by doctors' and nurses' has been shown to provide for a more comprehensive and effective relief of withdrawal complications (Kahan et al., 2004). For example, in a retrospective chart review at in the emergency department of two Toronto hospitals, Kahan et al., (2004) conducted a structured chart audit on 209 charts of patients with alcohol related problems. This study's purpose was to describe differences in how emergency departments manage alcohol withdrawal patients. The focus was on the documentation of withdrawal severity, use of symptom triggered benzodiazepine treatment, medications provided, ED length of stay and occurrences of seizures. One of the hospitals included in the study was a 350-bed community hospital, the second a 420bed hospital. Information was collected on demographics, chief complaints, length of stay, withdrawal severity, blood alcohol level, benzodiazepine dose administered, laboratory tests and consults ordered, discharge medication, and disposition. A standard 
audit was developed to clarify data definitions and review discrepancies to achieve ultimate reliability.

Kahan et al., (2004) noted that neither hospital used a standardized assessment tool to determine the severity of the withdrawal. The researchers found significant inconsistency between the two hospitals. At hospital A 48 patients received diazepam with no documented tremors and 20 patients were given diazepam with a BAL of greater than $35 \mathrm{mmol} / \mathrm{L}$. The researchers found that one hospital site administered significantly higher mean dose of diazepam (64mg vs $26 \mathrm{mg}, p<0.001)$, which was associated with a lower rate of withdrawal seizure activity (Kahan et al., 2004). Hospital A gave a total diazepam dose of $63.6 \mathrm{mg}$ and had only 1 associated seizure (0.8\%). In hospital B, a total of $25.9 \mathrm{mg}$ of diazepam was given, and 7 seizure occurrences (8.8\%) were found. Additionally, hospital A patients had a shorter emergency department length of stay ( $8 \mathrm{~h}$, $38 \mathrm{~min}, \mathrm{v} .10 \mathrm{~h}, 15 \mathrm{~min})$. This study demonstrates the variability in treatment and outcomes of patients experiencing alcohol withdrawal when a standardized protocol is not utilized. Researchers noted that findings of this study highlight the need for a standardized approach in symptom-triggered management of alcohol withdrawal.

A retrospective study by Duby, Berry, Ghayyem, Wilson, and Cocanour (2014) compared the outcomes of critical care patients experiencing alcohol withdrawal syndrome that have been treated with a symptom triggered protocol versus a non protocolized approach. One hundred and thirty-five study participants who were 18 years or older and admitted to an ICU for alcohol withdrawal were included. Patients were separated into two groups; one group was treated by physician preference and the other treated with escalating doses of benzodiazepines according to an AWS protocol. Length 
of ICU stay in the pre-protocol group was $9.6 \pm 10.5$ days and in the post-protocol group $5.2 \pm 6.4$ days $(p=0.0004)$. The post protocol group required less benzodiazepines $(\mathrm{p}=$ 0.0002). Ventilator days were decreased in the post-protocol group (5.6 \pm 13.19 vs $1.31 \pm$ 5.6 days, $p<0.0001)$. The need for intubation secondary to alcohol withdrawal was reduced in the post-protocol group $(22 \%$ vs $5 \%, p<0.001)$. These results demonstrate significant benefits in using a symptom-triggered protocol while treating alcohol withdrawal (Duby et al., 2014).

\section{Pharmacological treatments for Alcohol Withdrawal Syndrome}

Although there are no formal guidelines published, Mirijello et al. (2015) stated that a drug with a rapid onset, a long half-life, and one that is not metabolized in the liver would be the ideal drug for AWS. Current medications used in the treatment of AWS include benzodiazepines, phenobarbital, propofol, and dexmedetomidine.

Benzodiazepines such as lorazepam, chlordiazepoxide, oxazepam, and diazepam effect the central nervous system by causing sedation, hypnosis, and anticonvulsant activity. Phenobarbital is a barbiturate that is primarily used for the treatment of epilepsy by direct interaction with the GABA receptor. Propofol produces sedation and anxiolysis and is primarily used in cases of severe alcohol withdrawal. Dexmedetomidine produces the clinical effects of sedation, anxiolysis, analgesia, and sympatholysis.

In a recent systematic review by Schmidt et al. (2016), authors aimed to summarize the current literature pertaining to pharmacotherapy of severe alcohol withdrawal. A PubMed search was conducted to find articles relevant to the topic. They limited the search to articles from 1960-2015. Initially, 739 articles were retrieved. Two independent reviewers narrowed the research to 27 articles using a consensus decision for inclusion. 
The studies reviewed by Schmidt et al. (2016) provided insight into the pharmacological agents used in AWS. Benzodiazepines such as lorazepam, chlordiazepoxide, oxazepam, and diazepam effect the central nervous system by causing sedation, hypnosis, and anticonvulsant activity. Benzodiazepines as the first line treatment for AWS was suggested by a landmark study in 1969 (Kaim, Klett, \& Rothfeld, 1969). Kaim et al. (1969) randomized 500 patients to 1 of 4 different medications (chlordiazepoxide a benzodiazepine, hydroxyzine a sedating antihistamine, chlorpromazine an antipsychotic agent, thiamine a vitamin, or a placebo). Patients who were given the chlordiazepoxide had the lowest incidence of seizures or DTs which led to the establishment of benzodiazepines as the first-line treatment for alcohol withdrawal (Schmidt et al., 2016).

The systematic review by Schmidt et al. (2016) concluded there is lack of available research to guide management of AWS. These authors noted that although many other pharmacological treatments have been studied, the use of benzodiazepines remains the standard of care, diazepam being the most favorable (Schmidt et al., 2016). Additionally, Schmidt et al. (2016) noted that studies in which benzodiazepines were used in combination with phenobarbital, there was a reduction in length of stay for patients with AWS. Lastly, these authors concluded that the role of dexmedetomidine for treatment of AWS is unclear, but there may be a relationship between the use of dexmedetomidine and a decreasing need for benzodiazepines leading to a potential avoidance of mechanical ventilation (Schmidt et al., 2016).

Benzodiazepines. Benzodiazepines are allosteric modulators of GABAa, they bind specific sites on the GABAa receptor complex. GABA receptors and their subtypes 
are distributed throughout the central nervous system. Once a benzodiazepine binds to its site on the receptor there is an increased likelihood that the receptor will allow the passage of chloride ions through its membrane resulting in reduced excitability of the target cell (Baldwin et al., 2013)

As is the case with many drugs, benzodiazepines also have significant risks and side effects. Some of the adverse effects of this drug class include sedation, drowsiness, mental slowing, and anterograde amnesia, psychomotor effects, tolerance and dependence (Baldwin et al., 2013). Respiratory depression and decreased ventilatory response are additional adverse effects of benzodiazepine use that can lead to hypercapnia and increased hypopnea events (Ashton, 1994).

The mainstay of the pharmacological treatment in alcohol withdrawal is with benzodiazepines (Schuckit, 2014). Benzodiazepines are also used for a variety of disease states because they assist in anxiety relief, induction and maintenance of sleep, muscle relaxation, and prevention and cessation of seizures. There are a variety of benzodiazepines that differ in their potency, timing of effectiveness, and duration of action. Research has not found one specific benzodiazepine class to be superior to another (Schuckit, 2014). Specific benzodiazepine used for alcohol withdrawal is based largely on the pharmacokinetic abilities of that drug (Schuckit, 2014). Many treatment regimens include a combination of benzodiazepines with long half-lives, like diazepam, and those with shorter half-lives, like lorazepam (Schuckit, 2014).

To analyze the evidence for the efficacy and potential harmful effects of benzodiazepines, a metanalysis was performed by Holdbrook, Crowther, Lotter, Cheng, and King (1999). Articles were considered if they were randomized control trials (RCTs) 
that compared benzodiazepines to a placebo in patients experiencing acute alcohol withdrawal. MEDLINE and the Cochrane Controlled Trials Registry were searched, 23 trials were identified, 11 (total of 1,286 patients) of which met the inclusion criteria (total of 1,286 patients). Benzodiazepines used in these studies included chlordiazepoxide, diazepam, oxazepam, and lorazepam. The meta-analysis displayed the benefit of benzodiazepines in comparison to placebo $(\mathrm{OR}=3.28,95 \% \mathrm{CI}[1.30,8.28])$. Data comparison between benzodiazepines and other drugs could not be pooled and none of the alternative treatments were found to be more beneficial than benzodiazepines. No significant difference was found between benzodiazepines and alternative therapies in regard to adverse events (Holdbrook et al., 1999). Holdbrook et al. (1999) concluded that benzodiazepines should remain the gold standard for treatment of acute alcohol withdrawal.

Alternative treatments have been examined because of the concerns of central nervous depression with high doses of benzodiazepines. Although other therapies have been researched, many do not contain the anti-epileptic effect that benzodiazepines do. The effects benzodiazepines have on seizures and delirium have been thoroughly researched (Holdbrook et al., 1999). Holdbrook et al. (1999) stated that the most important consideration in withdrawal management is not which benzodiazepine to use, but to ensure that adequate and early doses are administered.

A systematic review (Amato, Minozzi, \& Davoli, 2011) examined the efficacy and safety of current pharmacological interventions used in alcohol withdrawal. The Cochrane Database of Systematic Reviews was searched, 114 studies met inclusion criteria. They reviewed five treatments including benzodiazepines, anticonvulsants, 
baclofen, GHB and psychotropic analgesic nitrous oxide (PAN). Outcomes assessed were alcohol withdrawal seizures, adverse events, and dropouts. Benzodiazepines performed better for seizures when comparing the five treatments to a placebo (95\% CI [0.004, 0.69]). When comparing the five treatments against one another, benzodiazepines performed better than antipsychotics for seizures (95\% CI $[0.07,0.88])$. The authors concluded that though alternative treatments for alcohol withdrawal carry less side effects, benzodiazepines display a protective benefit against seizures (Amato, Minozzi, \& Davoli, 2011).

While benzodiazepines have shown good effect in prevention of seizures, they can sometimes have negative effects on both cognitive and behavioral aspects. A systematic review article by Kok, Slooter, Hilegers, Dijk, and Veldhuijzen (2018) examined the role that benzodiazepines had on neuropsychiatric outcomes. The researchers identified 3,066 studies and surveyed them for inclusion criteria of ICU patients and 18 years or older. Forty-nine studies were ultimately included and consisted of randomized control trials, prospective cohort studies, retrospective cohort studies, and point prevalence studies.

The first outcome examined was the association between benzodiazepine use and adverse neuropsychiatric outcomes during hospitalization. Thirty-five of the included studies reported neuropsychiatric events either during hospitalization, after discharge, or both (Kok et al., 2018). Researchers defined neuropsychiatric events as delirium, posttraumatic stress disorder, depression, anxiety, and deficits in cognitive functioning. The Confusion Assessment Method (CAM) was the most frequently used tool for assessing patient's neuropsychiatric state. Kok et al. (2018) found 24 studies that identified 
benzodiazepine administration as a risk factor for delirium and two studies reported findings of anxiety, depression, or cognitive dysfunction post benzodiazepine administration.

The secondary outcome examined was benzodiazepine use and long-term neuropsychiatric outcomes. Fourteen studies reported long-term effects post benzodiazepine use, including symptoms of post-traumatic stress disorder (PTSD), depression, anxiety, and cognitive impairment. The symptoms were reported from the time of hospital discharge to 41 months after an ICU stay, with a median of 4.5 months (Kok et al., 2018). The cumulative dose and duration of midazolam and lorazepam use were acknowledged as a risk factor for neuropsychiatric impairment in four of the eight studies. Four studies displayed no correlation between benzodiazepine use and long-term neuropsychiatric outcomes. A large causal relationship was identified between benzodiazepines and delirium. Support for a noncausal association between benzodiazepines and long-term effects was apparent but small. This review suggests that benzodiazepine use in an ICU may contribute to development of neuropsychiatric outcomes (Kok et al., 2018).

In conclusion the primary treatment for alcohol withdrawal remains benzodiazepines, and these are administered using a front loading, fixed dose, or symptom triggered approach (Perry, 2014). Although benzodiazepines remain the leading choice due to their prevention of withdrawal and withdrawal-related seizures, they have also been associated with negative effects, including respiratory depression, over sedation and neuropsychiatric outcomes (Kok et al., 2018; Schmidt et al., 2016). Patients experiencing alcohol withdrawal that is refractory to high doses of benzodiazepines, may 
require the addition of alternative 'rescue' medications, such as phenobarbital, propofol, or dexmedetomidine.

Phenobarbital. Phenobarbital is frequently used for the treatment of alcohol withdrawal because of its synergistic effects when administered with benzodiazepines (Schmidt et al., 2016). Phenobarbital is used in patients who have proven to be refractory to high doses of benzodiazepines, meaning the patients' symptoms are still present even after accumulative diazepam doses of $>150 \mathrm{mg}$ or a lorazepam dose $>30 \mathrm{mg}$ (Schmidt et al., 2016). A phenobarbital dose of $1500 \mathrm{mg}$ to $2000 \mathrm{mg}$ is usually given on the first day the patient begins experiencing delirium (Schuckit, 2014).

Rosenson et al. (2012) conducted a prospective, randomized, double-blind, placebo-controlled study of emergency department patients with alcohol withdrawal syndrome. The researchers hypothesized that when a single dose of intravenous phenobarbital was combined with a symptom-guided lorazepam withdrawal protocol, a decrease in ICU admission rates would be seen. To be included in this study patients had to be older than 18, display symptoms of acute alcohol withdrawal, and have an anticipated need for admission. All participants were placed on the institutional symptom-guided lorazepam-based alcohol withdrawal protocol. Patients then were randomized to either receive a single dose of intravenous phenobarbital $(10 \mathrm{mg} / \mathrm{kg}$ in $100 \mathrm{~mL}$ of $\mathrm{NS}$ ) or $100 \mathrm{~mL}$ of normal saline. The primary outcomes measured were initial level of hospitalization from the emergency department, hospital length of stay, total amount of lorazepam used per patient, and incidence of adverse events. One hundred and ninety-eight patients enrolled, and 102 patients met the inclusion criteria established. 
Using randomization, fifty-one patients were placed in the phenobarbital group and 51 patients were placed in the placebo group (Rosenson et al., 2012).

Investigators found that patients who received a single dose of phenobarbital had a decreased ICU admission rate. In the phenobarbital group $8 \%$ had to be admitted to the ICU and in the placebo group $25 \%$ had to be moved to ICU, $95 \%$ CI [4, 32].

Phenobarbital also resulted in decreased use of continuous lorazepam infusion (4\% vs. $31 \%, 95 \% \mathrm{CI}[14,41])$ and decreased total lorazepam required (26 vs. $49 \mathrm{mg} ; 95 \% \mathrm{CI}$, [7, 40]) (Rosenson et al., 2012). There were no identified differences in floor ward admission, total hospital length of stay, incidence of adverse outcomes (including intubation, seizure, or mechanical restraints). Rosenson et al. (2012) concluded that when phenobarbital is administered in combination with lorazepam, incidence of ICU admission decreases.

Dexmedetomidine. Dexmedetomidine (Precedex) is an exceedingly selective $\alpha 2$ agonist that was approved by the U.S. Food and Drug Administration (FDA) as an ICU sedative in 1999 (Abramov, Nogid, \& Nogid, 2005). a2-Adrenoreceptors are found throughout the body in the central nervous system, peripheral nervous system, liver, pancreas, kidneys and eyes (Ungarian, Rankin, \& Then, 2019). It activates receptors in the medullary vasomotor center leading to a decrease in norepinephrine synthesis and sympathetic outflow (Schmidt et al., 2016). Sedation, anxiolysis, analgesia, and sympatholysis are all seen in the patient after administration of dexmedetomidine (DEX). It is a favorable drug in the ICU setting because of its preservation of respiratory function. Research related to this medication has shown no suppression of respiratory rate or gas exchange (Kaur \& Singh, 2011). 
Dexmedetomidine (DEX) is metabolized by the primarily the liver and excreted renally (Abramov et al., 2005). It is administered intravenously with an onset of action of approximately 15 minutes and reaches its peak concentration after one hour of continuous infusion (Ungarian et al., 2019). Patients with hepatic dysfunction may require lower doses but renally dosing dexmedetomidine is not indicated (Ungarian et al., 2019). Administration should be cautioned in patients who are hypovolemic, hypotensive, or elderly and in patients with advanced heart block, severe ventricular dysfunction, diabetes mellitus, or chronic hypertension (Abramov et al., 2005). This population is more at risk for pronounced hypotension and bradycardia due to DEX administration (Ungarian et al., 2019).

As with any medication, dexmedetomidine also has its reported side effects. Martin, Ramsay, Mantz, \& Sum-Ping, (2003) studied the adverse effects of dexmedetomidine. Martin et al. (2003) compared dexmedetomidine with a placebo in 401 postsurgical patients in an ICU double blind, randomized, multicenter control trial. The most common adverse effects of dexmedetomidine were hypotension in 61 of 203 patients $(30 \% ; p<.001)$, hypertension in 24 of 203 patients $(12 \% ; p=.005)$ and bradycardia in 18 of 203 patients $(18 \% ; p=.003)$ (Martin, Ramsay, Mantz, \& Sum-Ping, 2003).

In a randomized control trial that compared the use of dexmedetomidine and midazolam for sedation in ICU patients. Riker et al. (2009) conducted a prospective, double-blind, randomized control trial among 375 medical/surgical ICU patients on mechanical ventilation. Sedation and delirium levels were assessed using the Richmond Agitation-Sedation Scale (RASS) and the Confusion Assessment Method for the ICU 
(CAM). The intervention included an infusion of dexmedetomidine, or midazolam, titrated to achieve light sedation (RASS between -2 and +1 ). Outcomes assessed were as follows: percentage of time within target RASS, prevalence and duration of delirium, use of fentanyl and open label midazolam, and nursing assessments. Secondary outcomes included duration of mechanical ventilation, ICU length of stay, and adverse events.

Riker et al. (2009) found no significant difference in the percentage of time within the target RASS range between the two medications; $77.3 \%$ in the dexmedetomidine group versus $75.1 \%$ of time in the midazolam group (95\% CI, $[-3.3,7.5] ; p=.18)$. Prevalence of delirium was greater in midazolam treated patients (76.6\%) than the group of patients receiving dexmedetomidine (54\%) (95\% CI, [14, 33]; $p<0.001)$. Patients treated with dexmedetomidine had a shorter median time to extubation of 1.9 days (95\% CI, $[3.1,4.0])$ vs 5.6 days $(95 \% \mathrm{CI},[4.6,5.9] p=.01)$. Dexmedetomidine-treated patients also had shorter ICU lengths of stay (5.9 days (95\% CI, [5.7, 7.0]) vs 5.6 days (95\% CI, $[6.7,8.6] p=.24)$. When assessing for safety outcomes, patients treated with dexmedetomidine had greater incidence of bradycardia than those treated with midazolam ( $42 \%$ vs $18.9 \% ; p=<.001)$. Bradycardia was defined as a heart rate less than 40 beats per minute. Among the patients that experienced bradycardia, $4.9 \%$ of them required interventions such as infusion titration or interruption. Six patients treated with dexmedetomidine infusion required atropine to reverse bradycardia (Riker et al., 2009). Riker et al. (2009) concluded that dexmedetomidine and midazolam have equal efficacy in achieving targeted sedation levels in ventilated patients. Dexmedetomidine patients had an overall shorter time on the ventilator, in the ICU, and experienced less delirium. 
The most notable adverse effect was bradycardia, seen in the dexmedetomidine treated patients (Riker et al., 2009).

Dexmedetomidine use in Alcohol Withdrawal. Recent advances in research have shown that dexmedetomidine appears to be an effective adjunct that may diminish the hyperautonomic symptoms of alcohol withdrawal syndrome (VanderWeide et al., 2016). Dexmedetomidine provides a lighter level of sedation in comparison to other agents used as treatment for AWS. It has become promising in the management of AWS in combination with benzodiazepines, as it has little effect on respiratory depression. This drug is available as an intravenous infusion making it easier to use as an adjunctive therapy.

Dexmedetomidine produces a sedated but arousable state, and because of this it may reduce symptoms associated with AWS while decreasing the total dose of benzodiazepines used to sedate patients (Schuckit, 2014). Dexmedetomidine should not be used as a monotherapy for alcohol withdrawal because it lacks the GABA receptor activity required to prevent withdrawal-related seizures (Schmidt et al., 2016).

Beg et al. (2016) performed a retrospective cohort study of 77 patients admitted to an adult medical intensive care unit. This study was conducted at Kaiser Permanente Santa Clara Medical Center in their 30-bed intensive care unit. The objective identified was to assess the effect of dexmedetomidine on severe alcohol withdrawal and compare its use with solely benzodiazepines. They identified patients by using the ICD-9 codes of 291.0, 291.3 and 291.81. They excluded patients without confirmed alcohol withdrawal, those with ICU stays less than 20 minutes, those receiving dexmedetomidine for reasons other than withdrawal, and those with CIWA scores fewer than 5. The researchers 
grouped patients by whether they were treated with only benzodiazepines or a combination of benzodiazepines and dexmedetomidine (Beg et al., 2016). Primary outcomes measured was the difference in lorazepam equivalents and CIWA scores in the 24-hours before and after initiation of dexmedetomidine therapy.

Results displayed the frequency of dexmedetomidine used increased dramatically between $2009(16.7 \%)$ and $2013(82.4 \%)(p=0.01)$. Initiation of dexmedetomidine therapy was associated with significant improvements in CIWA scores over corresponding 24-hour intervals $(p<0.01)$. Benzodiazepine use was decreased in the dexmedetomidine group but was not statistically significant at 24 hours $(p=0.10)$. Dexmedetomidine was also associated with significantly longer hospitalizations $(p<$ 0.01). These results concluded that the use of dexmedetomidine initiation was associated with a reduction in short-term alcohol withdrawal symptoms patients in the intensive care unit. Beg et al., 2016 recommended that further research be conducted to evaluate the efficacy and cost effectiveness of dexmedetomidine in severe alcohol withdrawal (Beg et al., 2016).

Similarly, a single-center retrospective-controlled cohort analysis was used to evaluate early dexmedetomidine use in critically ill patients with AWS. VanderWeide et al., (2016) screened a total of 213 patients admitted to the ICU at the University of Colorado between September 15, 2008 and September 30, 2012. Data was collected through electronic and paper charts of those aged 18-80. Inclusion criteria was a recorded CIWA score $>8$ and administration of $>8 \mathrm{mg}$ of lorazepam within a six-hour window. Patients were excluded from the study if their symptoms were not severe enough (a CIWA score of $<8$ or received $<8 \mathrm{mg}$ of lorazepam), they were admitted to the ICU for 
reasons other than AWS, or they passed away within 72 hours of admission. Patients were separated into two groups; those who received dexmedetomidine and those who did not (control group) (VanderWeide et al., 2016). The primary outcome examined was the cumulative 12-hour benzodiazepine dose requirement change (post minus pre) in those who received dexmedetomidine compared to control patients who did not. Secondary outcomes included cumulative 24-hour benzodiazepine change (post minus pre), length of ICU and hospital stay, incidence and duration of mechanical ventilation, symptom severity using CIWA scores, average hemodynamic changes over 24 hours (post minus pre) and use of other adjunctive managements for AWS. Primary safety outcomes included incidence of hypotension and bradycardia (VanderWeide et al., 2016).

Of the 213 patients screened for inclusion criteria, 20 patients fit the criteria for the dexmedetomidine group, and 22 patients were placed in the control group (VanderWeide et al., 2016). The median time for dexmedetomidine administration from hospital admission was 26.1 hours (interquartile range [IQR] 15.1-32.7). Results showed the mean cumulative 12-hour benzodiazepine difference was $20 \mathrm{mg}$ ( $\mathrm{SD} \pm 22.3 \mathrm{mg}$ ) in the dexmedetomidine group, as compared to $8.3 \mathrm{mg}(\mathrm{SD} \pm 11.7 \mathrm{mg})$ in the control group $(p=$ .0455). The mean cumulative 24-hour benzodiazepine difference was $29.6 \mathrm{mg}$ (SD \pm $37.3)$ in dexmedetomidine versus $11 \mathrm{mg}(\mathrm{SD} \pm 21.7)$ in control group, $(p=0.06)$. Length of stay was not significantly different between the intervention group and control group (86.6 hours [IQR 57.5-137.1] vs. 54 hours [IQR 38.7-146]). Although dexmedetomidine demonstrated a decrease in length of intubation (24.9 hours versus 48.8 hours, $p=.049$ ), there were no significant differences noted in the $8(40 \%)$ patients receiving dexmedetomidine who required mechanical ventilation and the $9(41 \%)$ patients of the 
control group who required intubation $(p=1.0)$. Average CIWA score changes 24 hours pre and post dexmedetomidine administration was not statistically significant (2.67 vs 2.72 respectively). Safety outcomes displayed 7 incidences of bradycardia in the group receiving dexmedetomidine and zero in the control group $(p=<.01)$. The mean change in heart rate was decreased in patients receiving dexmedetomidine (17.9 bpm [SD \pm 16.0$]$ and control $2.8 \mathrm{bpm}[\mathrm{SD} \pm 11.8])$. Results of this study demonstrated a correlation between the use of dexmedetomidine and the decrease of 12-hour benzodiazepine requirements in patients experiencing alcohol withdrawal syndrome (VanderWeide et al., 2016).

\section{Conclusions}

The systematic review by Schmidt et al. (2016) concluded there is lack of available research to guide management of AWS. These authors noted that although many other pharmacological treatments have been studied, the use of benzodiazepines remains the standard of care, diazepam being the most favorable (Schmidt et al., 2016). The ideal treatment is a symptom-triggered protocol that includes an increasing dose of benzodiazepines that coincides with symptom severity. Additionally, Schmidt et al. (2016) noted that studies in which benzodiazepines were used in combination with phenobarbital, there was a reduction in length of stay for patients with AWS. Lastly, these authors concluded that the role of dexmedetomidine for treatment of AWS is unclear, but there may be a relationship between the use of dexmedetomidine and a decreasing need for benzodiazepines leading to a potential avoidance of mechanical ventilation (Schmidt et al., 2016). 
In conclusion, performing additional research regarding the use of dexmedetomidine for the treatment of withdrawal will have promising benefits for the outcomes of many patients suffering from alcoholism. Alcohol withdrawal syndrome and its treatment has been well studied, along with the pharmacological use of benzodiazepines. In order to improve the care of this vulnerable population, dexmedetomidine as an adjunctive therapy must be thoroughly researched. 


\section{Theoretical Framework}

Nursing and medicine rely on symptomology to guide practice and research. Symptoms are the perceived indicators of a significant change in the normal functioning of the human body. Symptoms serve as red flags for the health care provider to identify, diagnose, and treat patients. Symptoms are the central focus of the Theory of Unpleasant Symptoms (TUS).

The Theory of Unpleasant Symptoms (TOUS) was developed in 1995 and has been used to guide research for years. This theory was developed to enhance understanding of the relationship between a variety of symptoms and the symptom experience to in turn, manage these unpleasant symptoms using effective interventions. Because the most common reason that patients seek health care is because they are experiencing unpleasant symptoms, understanding such symptoms is imperative. This middle range theory was developed with the assumptions that there are commonalities among symptoms that can explain and guide research (Lenz, Pugh, Milligan, Gift, \& Suppe, 1997). The theory of unpleasant symptoms proposes that the factors associated with any given symptom may influence a variety of concurrent symptoms and that a single event may result in a multitude of symptoms. Therefore, the use of therapeutic interventions aimed at lessening the severity of one symptom, may consequently be effective in relieving more than one symptom.

There are three major components involved in the theory of unpleasant symptoms: the symptoms that the individual is experiencing, the influencing factors that give rise to or affect the nature of the symptom experience, and the consequences of the symptom experience (Lenz et al.). Symptoms are described in dimensions of intensity, timing, level of perceived distress, and quality. The Theory of Unpleasant Symptoms 
identified three categories of variables that influence these dimensions. These three variables include: physiologic factors, psychologic factors, and situational factors. The three categories of factors are intertwined and may interact with each other to influence the symptom experience. The final component of TOUS is performance, the outcome or effect of the symptom experience (Lenz, et al.). TOUS identifies a reciprocal relationship between the three components.

With the multiple symptoms associated with alcohol withdrawal, the theory of unpleasant symptoms will lend itself to the research question at hand. Middle-range theories guide nursing research by helping to develop theoretically derived measurement instruments that map the various dimensions of symptoms. TOUS is valuable in individualizing symptom management because it forces the researcher to confront the fact unidimensional measurement of unpleasant symptoms is not realistic. The Theory of Unpleasant Symptoms forces nursing to look at symptoms and symptom management as a multidimensional phenomenon.

The data being collected for the purpose of determining if dexmedetomidine safely decreases the total dose of benzodiazepines administered to patients experiencing alcohol withdrawal is largely based on symptoms. Alcohol withdrawal is treated based on a series of symptoms displayed by the patient. In their state of autonomic hyperactivity, the provider will administer benzodiazepines in hopes to control the agitation and prevent seizures. By using the theory of unpleasant symptoms to guide this research, insight will be provided regarding the symptomology of Alcohol Withdrawal Syndrome. These symptoms, however, should be differentiated from the symptoms associated with large doses of benzodiazepines Understanding the difference between these two will help 
health care providers use a multifaceted approach when treating alcohol withdrawal. This study focused on the use of dexmedetomidine (Precedex) for the treatment of symptoms seen with alcohol withdrawal and its effect on the reduction of benzodiazepine dosing. 


\section{Methods}

\section{Purpose}

The purpose of this project was to determine if the use of dexmedetomidine impacts the dosage of benzodiazepines administered to patients admitted to the intensive care unit with severe alcohol withdrawal.

\section{Design}

A retrospective chart review was conducted to explore the relationship between dexmedetomidine use and the total administered dose of benzodiazepines in patients admitted to a medical intensive care unit with severe alcohol withdrawal. A CIWA-Ar score greater than 15 points corresponds with severe withdrawal syndrome and increased risk of progressing to delirium tremens and seizures. Severe alcohol withdrawal was also considered when symptoms of delirium tremens are present: coarse tremor, agitation, fever, tachycardia, profound confusion, delusions, and hallucinations. Signs of ketoacidosis, convulsions, and circulatory collapse may develop.

This study employed a two-group comparative chart review. Group one (control), included those treated with the standard of care alone and group two (intervention) included those treated with dexmedetomidine in addition to the standard of care.

\section{Site}

This research study was conducted at a single 247-bed, teaching, acute-care hospital in Rhode Island. The hospital has a 16-bed Intensive Care Unit.

\section{Sample}

A convenience sample was used in this study to recruit as many patients from the accessible population July 1, 2017 to July 1, 2019 who meet the eligibility criteria defined. Inclusion criteria was adults, greater than the age of 18; intensive care unit 
patients admitted for more than 24 hours with the ICD-9 diagnosis code for alcohol withdrawal; treatment group received dexmedetomidine within 72 hours of ICU admission. There were 68 charts selected for review. Of those, 40 charts were included in this study, 18 in group one (standard of care) and 22 charts in group two (dexmedetomidine group). A total of 28 charts were excluded from this study, 10 of which were deceased and 18 did not have alcohol withdrawal as a primary diagnosis. Patients were excluded from this study if the primary reason for their intensive care unit admission was not related to alcohol withdrawal syndrome or if the severity of withdrawal was a CIWA score of $<8$. A CIWA score of less than $<8$ requires little to no treatment and therefore were unusable in this study. Patients who received dexmedetomidine for alcohol withdrawal starting after 72 hours from admission were excluded. Pregnant patients, ICU stay less than 24 hours, or death within 72 hours from ICU admission were also be excluded.

\section{Procedures}

Approval from the investigational review boards of Rhode Island College and Lifespan were obtained for this research study. Permission was additionally obtained from the Chief Nursing Officer at the study site. The critical care attending physicians at the site, as well as the Intensive Care Unit's Clinical Manager were also notified.

Data was collected for patients from July 1, 2017 to July 1, 2019. The Information Systems (IS) LifeChart team at The Hospital site was contacted via a report request to extract data through the LifeChart (Epic) Clarity Database for the detailed data points based on the study population. Documentation that the IS LifeChart team retrieved included: 
- Primary outcome: Total benzodiazepine requirement during ICU stay and dexmedetomidine length of use.

- Secondary outcomes: Length of ICU stay

- Primary safety outcomes: Bradycardia $(\mathrm{HR}<60)$, and fever (temperature $>101^{\circ}$ ), incidence of mechanical ventilation

The medical records of potential subjects were reviewed by the researcher for inclusion and exclusion criteria. The records that met inclusion criteria were reviewed further to extract the identified data. No patient identifiers were collected. Data was reviewed with the IS LifeChart team during regular office hours and entered into an Excel spreadsheet. Confidentiality of data was maintained throughout the course of this study. Data retrieved was kept on an encrypted flash drive, using a password protected computer. All data was destroyed upon completion of the study's evaluation.

\section{Measurement}

A data collection tool was designed by the student researcher based on the literature and clinical experience. This data collection tool was designed using an excel spreadsheet, based on the key study variables and was submitted to the primary investigator (PI) for approval prior to use.

\section{Data analysis}

Descriptive statistics were used to examine the study variables and were summarized by using means and standard deviations. 


\section{Results}

A total of 68 charts were reviewed for inclusion criteria. Of those 68 charts, 28 charts did not meet inclusion criteria. A total of 40 charts met inclusion criteria and were included in this study. Group one $(n=18)$ included those admitted to the intensive care unit with alcohol withdrawal and treated with the standard of care alone. Group two $(n=22)$ included those admitted to the ICU with alcohol withdrawal treated with the standard of care and dexmedetomidine. Table 1 summarizes the data collected in both Group A and Group B.

Data collected for both Group one and Group two included age, ICU admission date, ICU length of stay in hours (depicted in Figure 3), total dose of Ativan administered during ICU stay (depicted in Figure 1), total dose of phenobarbital during ICU stay (depicted in Figure 2), dexmedetomidine use and duration, incidence of mechanical ventilation, bradycardia, and hyperthermia. The total, range, and mean of all the data was computed for all categories of data and compared between the two groups.

The age range of Group one was between the ages of 34 and 65 with the mean age being 48. The Ativan requirement for group one ranged from $14 \mathrm{mg}$ to $118 \mathrm{mg}$ with a mean of $59.8 \mathrm{mg}$. The total amount of Ativan administered for all the patients in Group one was $718 \mathrm{mg}$. The Phenobarbital requirements in Group one ranged from $0 \mathrm{mg}$ to 890 $\mathrm{mg}$ with a mean of $425.5 \mathrm{mg}$. The total amount of Phenobarbital administered for the patients in Group one was 5,107 mg. The overall length of ICU stay in Group one ranged from 33 hours to 102 hours with a mean of 57.8 hours. Incidence of bradycardia occurred once $(n=1)$ as well as hyperthermia $(n=1)$ in Group one. Group one had no patients requiring mechanical ventilation. 
The age range in Group two was 31-73 with a mean age of 53. Group two received a mean Ativan dose of $98 \mathrm{mg}$ ranging from $0 \mathrm{mg}$ to $288 \mathrm{mg}$. The total phenobarbital requirements in Group two ranged from $0 \mathrm{mg}$ to 2,343 $\mathrm{mg}$ with a mean of $865.9 \mathrm{mg}$. Overall in Group two there was a mean ICU length of stay of 120.4 hours, ranging between a minimum of 34 hours to a maximum of 195 hours. Incidence of bradycardia occurred in 10 patients $(n=10)$ in Group two. Incidence of hyperthermia occurred in 7 patients $(n=7)$ in Group two. Lastly, 7 patients $(n=7)$ required mechanical ventilation in Group two. Table one summarizes the data collected in both Group one and Group two. 
Table 1. Comparison between Group one and Group two

\begin{tabular}{|c|c|c|}
\hline & $\begin{array}{c}\text { Group One } \\
\text { (Standard of Care) } \\
n=18\end{array}$ & $\begin{array}{c}\text { Group Two } \\
\text { (Precedex and Standard of } \\
\text { Care) } \\
n=22\end{array}$ \\
\hline Age (y/o) & $34-64(\mathrm{M}=48)$ & $31-73(\mathrm{M}=53)$ \\
\hline $\begin{array}{l}\text { Benzodiazepine } \\
\text { Requirement } \\
\text { (Ativan in } \mathrm{Mg} \text { ) }\end{array}$ & $\begin{array}{l}\text { Total }=718 \mathrm{mg} \\
M=59.8 \mathrm{mg} \\
\text { Max }=118 \mathrm{mg} \\
\text { Min }=14 \mathrm{mg}\end{array}$ & $\begin{array}{l}\text { Total }=1,200 \mathrm{mg} \\
\mathrm{M}=98 \mathrm{mg} \\
\text { Max }=288 \mathrm{mg} \\
\text { Min }=0 \mathrm{mg}\end{array}$ \\
\hline $\begin{array}{l}\text { Barbiturate } \\
\text { Requirement } \\
\text { (Phenobarbital } \\
\text { in Mg) }\end{array}$ & $\begin{array}{l}\text { Total }=5,107 \mathrm{mg} \\
\mathrm{M}=425.5 \mathrm{mg} \\
\text { Max }=890 \mathrm{mg} \\
\text { Min }=0 \mathrm{mg}\end{array}$ & $\begin{array}{l}\text { Total }=550 \mathrm{mg} \\
M=865.9 \mathrm{mg} \\
M a x=2,343 \mathrm{mg} \\
\min =0 \mathrm{mg}\end{array}$ \\
\hline ICU LOS & $\begin{array}{l}\text { Total }=694 \mathrm{hrs} \\
\mathrm{M}=57.8 \mathrm{hrs} \\
\mathrm{Max}=102 \mathrm{hrs} \\
\mathrm{Min}=33 \mathrm{hrs}\end{array}$ & $\begin{array}{l}\text { Total }=1,387 \mathrm{hrs} \\
\mathrm{M}=120.4 \mathrm{hrs} \\
\text { Max }=195 \mathrm{hrs} \\
\operatorname{Min}=34 \mathrm{hrs}\end{array}$ \\
\hline $\begin{array}{l}\text { Bradycardia } \\
(H R<60)\end{array}$ & $\mathrm{n}=1$ & $\mathrm{n}=10$ \\
\hline $\begin{array}{l}\text { Hyperthermia } \\
\text { (Temp > 101) }\end{array}$ & $\mathrm{n}=1$ & $\mathrm{n}=7$ \\
\hline $\begin{array}{l}\text { Mechanical } \\
\text { Ventilation }\end{array}$ & $\mathrm{n}=0$ & $\mathrm{n}=7$ \\
\hline
\end{tabular}


Figure 1. Comparison of Mean Ativan Requirements between Group One and Two

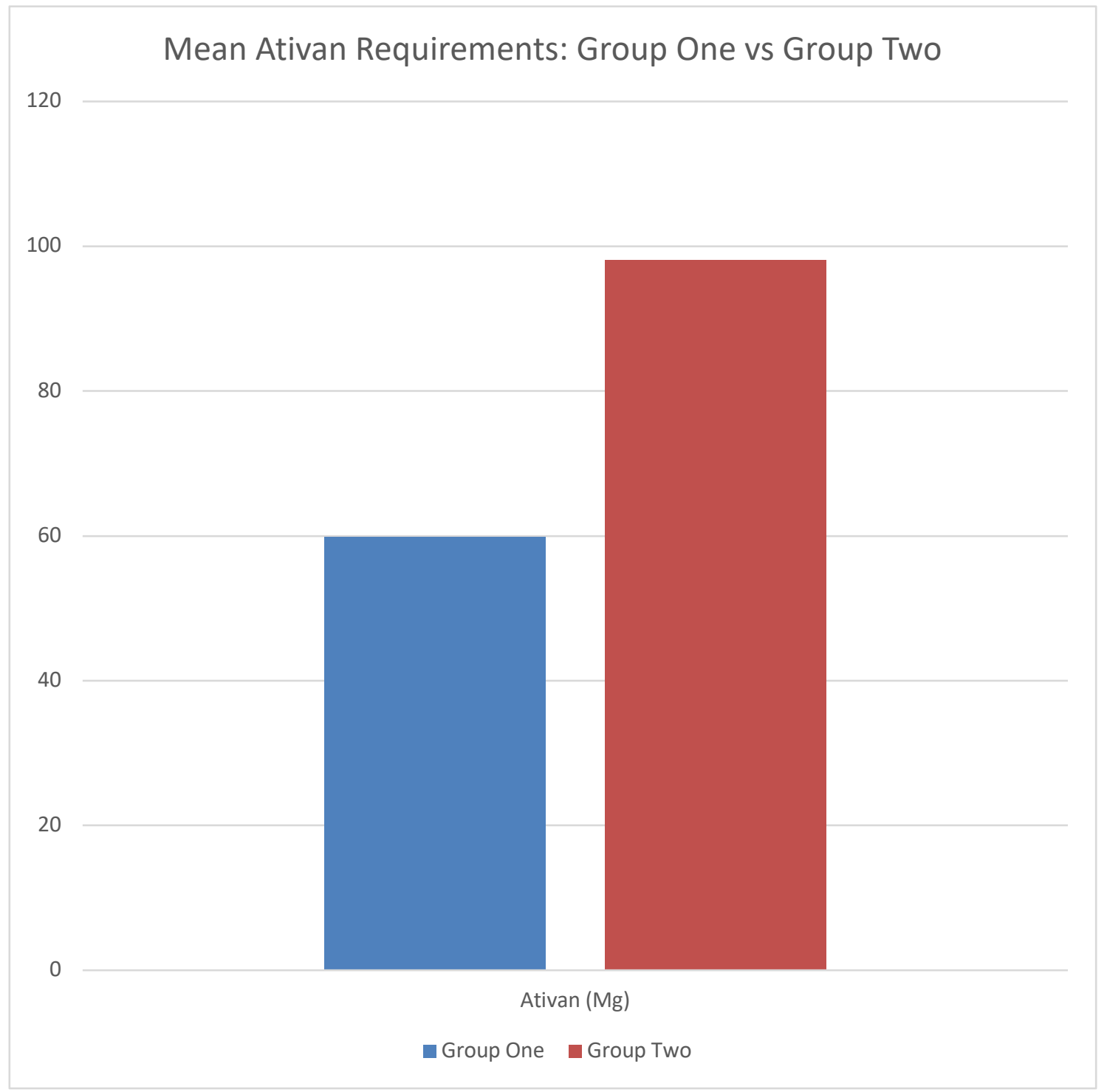


Figure 2. Comparison of Mean Phenobarbital Requirements between Group One and Two

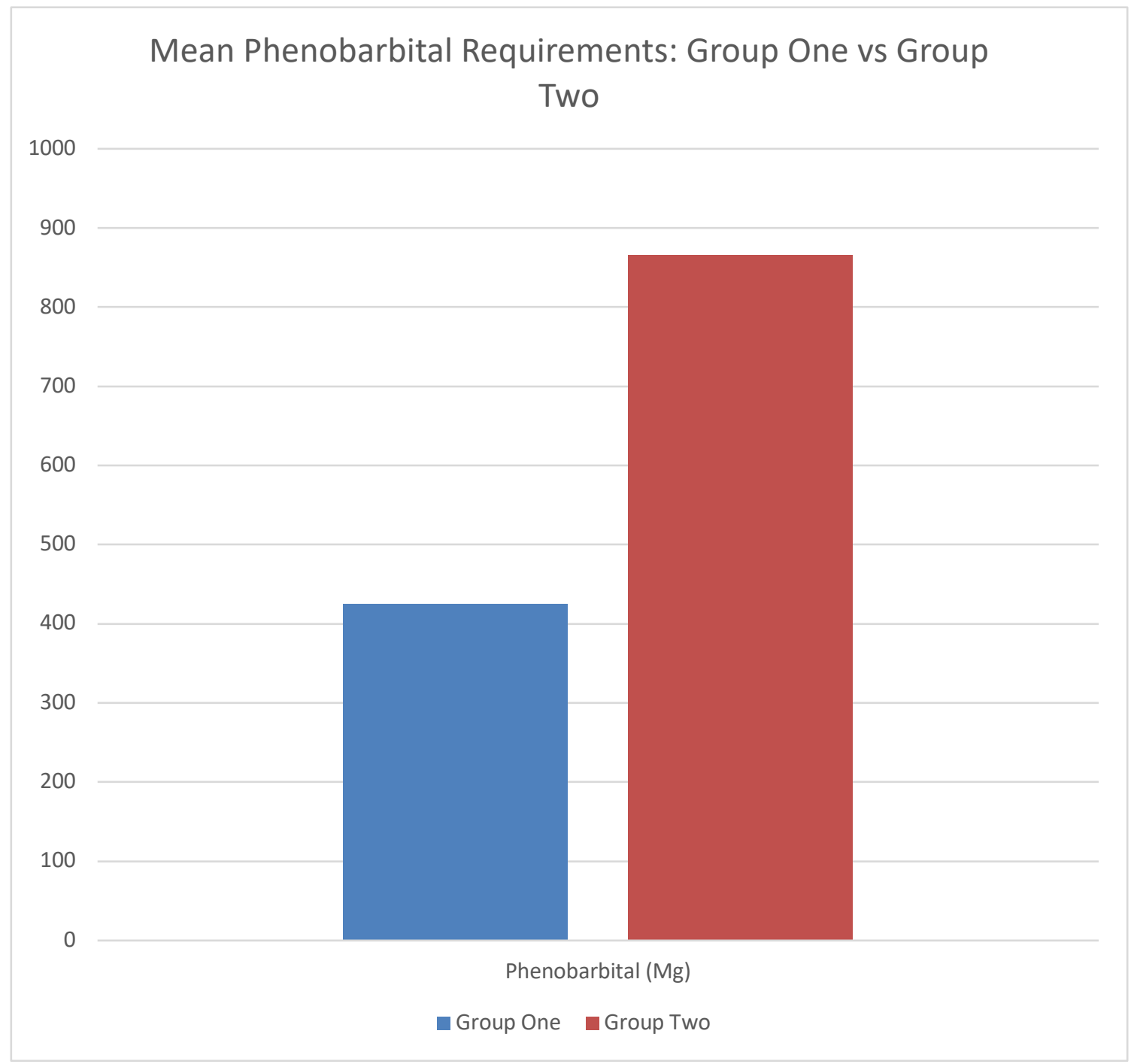


Figure 3. Comparison of Mean ICU Length of Stay between Group One and Two

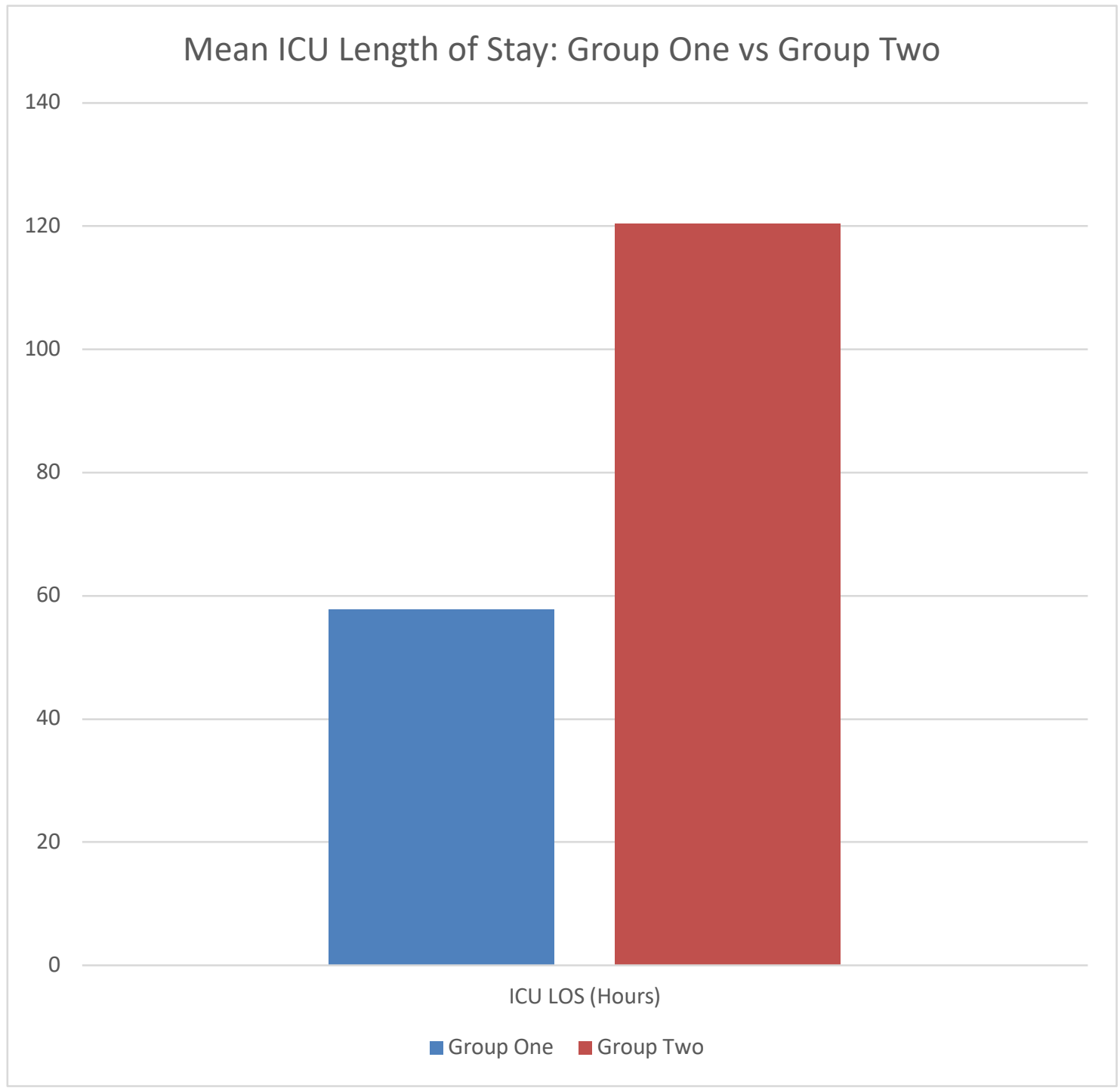




\section{Summary and Conclusions}

The use of large doses of benzodiazepines can lead to over sedation and respiratory depression (VanderWeide et al., 2016). Prolonged length of stay, increased risk of infections, delirium, and need for mechanical ventilation have all been reported as complications occurring in patients experiencing alcohol withdrawal (Sutton \& Jutel, 2016). A systematic review by Schmidt et al. (2016) concluded there is a lack of available research to guide management of alcohol withdrawal syndrome. Although benzodiazepines remain the standard of care, there is increasing use of dexmedetomidine in the clinical setting for the management of alcohol withdrawal (Schmidt et al., 2016).

The purpose of this project was the complete a retrospective chart review to evaluate if the use of dexmedetomidine reduces the quantity of benzodiazepines administered to patients experiencing alcohol withdrawal syndrome in an intensive care unit. This study was guided by The Theory of Unpleasant Symptoms (Lenz, Pugh, Milligan, Gift, \& Suppe, 1997). A sample of randomly selected patients admitted to the intensive care unit for alcohol withdrawal were separated into two groups. Group one consisted of patients that underwent alcohol withdrawal and were treated with the standard of care alone. Group two was patients that were treated with the standard of care along with the addition of dexmedetomidine. The researcher designed a data collection tool based on literature and clinical experience which included patient age, ICU length of stay, Ativan requirements, phenobarbital requirements, dexmedetomidine use and duration, and the incidence of bradycardia, hyperthermia, and mechanical ventilation.

Group one had a final sample size of 18 patients $(\mathrm{n}=18)$ and group two had a final sample size of 22 patients $(n=22)$. The mean benzodiazepine requirement was less in group one than it was in group two, $59.8 \mathrm{mg}$ vs $98 \mathrm{mg}$ respectively. Barbiturate 
requirement in group one was also less than group two, $425.5 \mathrm{mg}$ vs $865.9 \mathrm{mg}$ respectively. The mean ICU length of stay in group one was 57.8 hours and the mean ICU length of stay in group two was 120.4 hours. The incidence of bradycardia occurred once in group one, while it occurred 10 times in group two. Hyperthermia occurred once in group one and 7 times in group two. Lastly, the incidence of mechanical ventilation did not occur in group one while occurring 7 times in group two.

These findings indicate that the addition of dexmedetomidine was not associated with a decrease in total benzodiazepine requirement during acute alcohol withdrawal. The patients in group two had longer lengths of ICU stays, increased incidence of mechanical ventilation, increased bradycardia, and more hyperthermia. It is important to note that these findings were associations rather than causation. The chart review used in this project, however, also indicates that currently dexmedetomidine is only being initiated on patients who are refractory to large quantities of Ativan and phenobarbital. This raises the question: How would results differ if dexmedetomidine was started earlier in the patient's course of withdrawal?

This study was limited by factors such as incomplete or missing records, overall sample size, and limited eligibility to the exclusion criteria. This study was also limited by restrictions in data collection. The results may have been altered if the researcher had collected benzodiazepine and barbiturate administration from time of entering the emergency department rather than just during ICU stay.

In summary, the data collected from this study did not support an overall decrease in benzodiazepine requirement when dexmedetomidine was utilized for severe alcohol withdrawal in an intensive care unit. More research needs to be conducted on how to 
effectively treat and management alcohol withdrawal syndrome in the critically ill while minimizing the effects of large quantities of benzodiazepines. 


\section{Recommendations and Implications for Advanced Nursing Practice}

Alcohol withdrawal syndrome is a condition seen by intensive care units every day. Whether the patient presents specifically for alcohol withdrawal or undergoes alcohol withdrawal secondary to cessation for another illness. Complication alcohol withdrawal is not only difficult to manage but it can increase healthcare costs if not treated appropriately. It is imperative for providers to base care on evidence-based guidelines and recommendations. Continued Advanced Practice Provider (APP) led research regarding the use of dexmedetomidine is needed to determine its effectiveness in the treatment of severe alcohol withdrawal. This is consistent with recommendations made in the literature for further investigation to evaluate the efficacy and cost effectiveness of dexmedetomidine in alcohol withdrawal (Beg et al., 2016).

The Advanced Practice Registered Nurse (APRN) has the ability to present research to hospital administration and help develop policies and procedures regarding alcohol withdrawal syndrome. Perhaps, a randomized control trial in which dexmedetomidine was implemented earlier would produce alternative results.

The APRN has a unique opportunity to represent the profession of nursing and has a great impact on patient outcomes. They are able to educate other providers and nurses on the evidence regarding alcohol withdrawal treatment. The understanding of alcohol withdrawal syndrome and its effective treatments by APRNs is important for positive patient outcomes. 


\section{References}

Abramov, D., Nogid, B., \& Nogid, A. (2005, March). The role of Dexmedetomidine (Precedex) in the sedation of critically ill patients. Drug Forecast, 30, 158-161. Retrieved from

Amato, L., Minozzi, S., \& Davoli, M. (2011, June 15). Efficacy and safety of pharmacological interventions for the treatment of the alcohol withdrawal syndrome. Cochrane Drugs and Alcohol Group. https://doi.org/10.1002/14651858.CD008537.pub2

American Psychiatric Association. (2013). Diagnostic and statistical manual of mental disorders (5th ed.). Washington, DC: American Psychiatric Association.

Ashton, H. (1994). Guidelines for the rational use of benzodiazepines. Clinical Psychopharmacology.

Awissi, D., Lebrun, G., Coursin, D., Riker, R., \& Skrobik, Y. (2013). Alcohol withdrawal and delirium tremens in the critically ill: a systematic review and commentary. Intensive Care Medicine, 39, 17-30. http://dx.doi.org/10.1007/s00134-012-2758-y

Baldwin, D. S., Aitchison, K., Bateson, A., Curran, V., Davies, S., Leonard, B., ... Wilson, S. (2013). Benzodiazepines: risks and benefits. A reconsideration. Journal of Psychopharmacology, 27, 968-971. https://doi.org/10.1177/0269881113503509

Beg, M., Fisher, S., Siu, D., Rajan, S., Troxell, L., \& Liu, V. X. (2016). Treatment of alcohol withdrawal syndrome with and without dexmedetomidine. The Permanente Journal, 20, 49-53. https://doi.org/10.7812/TPP/15-113 
Center for Substance Abuse Treatment. Detoxification and Substance Abuse Treatment. . (2006). Screening and Assessment Instruments. Retrieved from https://www.ncbi.nlm.nig.gov/books/NBK64117/

DeCarolis, D. D., Rice, K. L., Ho, L., Willenbring, M. L., \& Cassaro, S. (2007). Symptom-driven lorazepam protocol for treatment of severe alcohol withdrawal delirium in the intensive care unit, 27, 510-518.

Dixit, D., Endicott, J., Burry, L., Ramos, L., Yeung, S. Y., Devabhakthuni, S., ... Bulloch, M. N. (2016). Management of acute alcohol withdrawal syndrome in critically ill patients. Pharmacotherapy. https://doi.org/10.1002/phar.1770

Duby, J. J., Berry, A., Ghayyem, P., Wilson, M. D., \& Cocanour, C. S. (2014, February 15). Alcohol withdrawal syndrome in critically ill patients: protocolized versus nonprotocolized management. Trauma Acute Care Surgery, 77, 938-943. https://doi.org/10.1097/TA.0000000000000352

Eberly, M., Lockwood, A., Lockwood, S., \& Davis, K. (2016, Oct). Outcomes after implementation of an alcohol withdrawal protocol at a single institution. Hospital Pharmacy. https://doi.org/10.1310/hpj5109-752

Eyer, F., Schuster, T., Felgenhauer, N., Pfab, R., Strubel, T., Saugel, B., \& Zilker, T. (2011, July). Risk assessment of moderate to severe alcohol withdrawalpredictors for seizures and delirium tremens in the course of withdrawal. Alcohol and Alcoholism, 46(4), 427-433. https://doi.org/10.1093/alcalc/agr053

Foy, A., Kay, J., \& Taylor, A. (1997). The course of alcohol withdrawal in a general hospital [Entire issue]. General Medicine and Alcohol \& Drug Units, 90. Retrieved from http://ovidsp.tx.ovid.com/sp-3.26.1a/ovidweb.cgi 
Frazee, E. N., Personett, H. A., Leung, J. G., Nelson, S., Dierkhising, R. A., \& Bauer, P. R. (2014). Influence of dexmedetomidine therapy on the management of severe alcohol wihtdrawal syndrome in critically ill patients. Journal of Critical Care, 29(11), 298-302. https://doi.org/10.1016/j.jcrc.2013.11.0016

Gold, J., Rimal, B., Nolan, A., \& Nelson, L. (2007). A strategy of escalating doses of benzodiazpines and phenobarbital administration reduces the need for mechanical ventilation in delirium tremens. Critical Care Medicine, 35, 724-730.

Grossman, S. C., \& Porth, C. M. (2014). Porth's pathophysiology: Concepts of altered health states (9th ed.). Philadelphia, PA: Lippincott Williams \& Wilkins.

Guirguis, E., Richardson, J., Kuhn, T., \& Fahmy, A. (2017). Treatment of severe alcohol withdrawal: A focus on adjunctive agents. Journal of Pharmacy Technology, 33, 204-212. https://doi.org/10.1177/8755122517714491

Holdbrook, A. M., Crowther, R., Lotter, A., Cheng, C., \& King, D. (1999). Meta-analysis of benzodiazepine use in the treatment of acute alcohol withdrawal. Canadian Medical Association, 160, 649-655. Retrieved from http://www.cmaj.ca/content/cmaj/160/5/649.full.pdf

Jastrzebska, I., Zwolak, A., Szczyrek, M., Wawryniuk, A., Skrzydlo-Radomanska, B., \& Daniluk, J. (2016, June 8). Biomarkers of alochol misues: recent advances and future prospects. Gastroenterology Review. https://doi.org/10.5114/pg.201660252

Jesse, S., Brathen, G., Ferrara, M., Keindl, M., Ben-Menachem, E., Tanasescu, R., ... Ludolph, A. C. (2016, September 1). Alcohol withdrawal syndrome: mechanisms, manifestations, and management. Acta Neurologica Scandinavica, 135, 4-16. https://doi.org/10.1111/ane.12671 
John, B. (2018, July 12). An integrative review of the theory of unpleasant symptoms. Journal of Advanced Nursing, 947-961. https://doi.org/10.1111/jan.13906

Kahan, M., Borgundvaag, B., Midmer, D., Borsoi, D., Edwards, C., \& Ladhani, N. (2004, February 14). Treatment variability and outcome differences in the emergency department management of alcohol withdrawal. EM Advances, 87-92. Retrieved from

Kaim, S. C., Klett, C. J., \& Rothfeld, B. (1969, June). Treatment of the acute alcohol withdrawal state: a comparison of four drugs. American Journal of Psychiatry, 125(12), 1640-1646. Retrieved from https://pubs.niaaa.nih.gov/publications/ahrw19-1/34-35.pdf

Kaur, M., \& Singh, P. (2011, Jul). Current role of dexmedetomidine in clinical anesthesia and intensive care. Anesthesia Essays and Researches, 128-133. https://doi.org/10.4103/0259-1162.94750

Kok, L., Slooter, A. J., Hilegers, M. H., Dijk, D. V., \& Veldhuijzen, D. S. (2018, October). Benzodiazepine use and neuropsychiatric outcomes in the ICU: A systematic review. Critical Care Medicine, 46, 1674-1680. https://doi.org/10.1097/CCM.0000000000003300

Lenz, E., Pugh, L., Milligan, R., Gift, A., \& Suppe, F. (1997, March). The Middle-Range Theory of Unpleasant Symptoms: An Update. Advances in Nursing Science, 19. Retrieved from http://ovidsp.tx.ovid.com.ric.idm.oclc.org

Linn, D., \& Loeser, K. (2015). Dexmedetomidine for alcohol withdrawal syndrome. Annals of Pharmacotherapy, 49, 1336-1342. https://doi.org/10.1177/10600280156007038 
Littlefield, A. J., Heavner, M. S., Eng, C. C., Cooper, D. A., Heavner, J. J., Kurtz, J. M., \& Pisani, M. A. (2018, July). Correlation between mMINDS and CIWA-Ar scoring tools in patients with alcohol withdrawal syndrome. American Journal of Critical Care Medicine, 27. https://doi.org/10.4037/ajcc2018547

Littlefield, A. J., Heavner, M. S., Eng, C. C., Cooper, D. A., Heavner, J. K., Kurtz, J. M., \& Pisani, M. A. (2018, July). Correlation between mMINDS and CIWA-Ar scoring tools in patients with alcohol withdrawal syndrome. American Journal of Critical Care, 27(4). https://doi.org/10.4037/ajcc2-18547

Ludtke, K. A., Stanley, K. S., Yount, N. L., \& Gerkin, R. D. (2015, March). Retrospective review of critically ill patients experiencing alcohol withdrawal: dexmedetomidine versus propofol and/or lorazepam continuous infusions. Hospital Pharmacy. https://doi.org/10.1310/hpi5003-208

Marc, S. (2014, November 27). Recognition and management of withdrawal delirium. The New England Journal of Medicine. https://doi.org/10.1056/NEJMra1407298

Martin, E., Ramsay, G., Mantz, J., \& Sum-Ping, S. (2003, Jan 18). The role of the alpha2adrenoceptor agonist dexmedetomidine in postsurgical sedation in the intensive care unit. Journal of Intensive Care Medicine, 29-41. https://doi.org/10.1177/0885066602239122

McEwen, M., \& Wills, E. M. (2014). Theoretical basis for nursing (4th ed.). Philadelphia, PA: Lippincott Williams \& Wilkins. Mirijello, A., D’Angelo, C., Ferrulli, A., Vassallo, G., Antonelli, M., Caputo, F., ... Addolorato, G. (2015, Februray 10). Identification and Management of Alcohol 
Withdrawal Syndrome. Therapy in Practice. https://doi.org/10.1007/s40265-0150358

Myrick, A. H. (1998). Treatment of alcohol withdrawal. Alcohol Heath \& Research World, 22(1). Retrieved from http://web.b.ebscohost.com.ric.idm.oclc.org National Institute on Alcohol Abuse and Alcoholism. (2017). www.niaaa.nih.gov

Perry, E. (2014). Inpatient management of acute alcohol withdrawal syndrome. Therapy in Practice, 28, 401-410. https://doi.org/10.1007/s40263-014-0163-5

Riker, R. R., Shehabi, Y., Bokesch, P. M., Ceraso, D., Wisemandle, W., Koura, F., ... Douglas, B. (2009, February 4). DExmedetomidine vs midazolam for sedation of critically ill patients. Caring for the critically ill patients, 301(5), 489-499. https://doi.org/10.1001/jama.2009.56

Rosenson, J., Clements, C., Simon, B., Vieaux, J., Graffman, S., Vahidnia, F., ... Alter, H. (2012, January 4). Phenobarbital for acute alochol withdrawal: a prospective randomized double-blind placebo-controlled study. The Journal of Emergency Medicine, 44, 592-598. https://doi.org/10.1016/j.jemermed.2012.07.056

Schmidt, K. J., Doshi, M. R., Holzhausen, J. M., Natavio, A., Cadiz, M., \& Winegardner, J. E. (2016). Treatment of severe alcohol withdrawal. Annals of Pharmacotherapy, 50, 389-401. http://dx.doi.org/10.1177/1060028016629161

Schmidt, K. J., Doshi, M. R., Holzhausen, J. M., Natavio, A., Cadiz, M., \& Winegardner, J. E. (2016). Treatment of severe alcohol withdrawal. Annals of Pharmacotherapy, 50, 389-401. https://doi.org/10.1177/1060028016629161 
Schuckit, M. A. (2014, November 27). Recognition and management of withdrawal delirium. New England Journal of Medicine. https://doi.org/10.1056/NEJMra1407298

Schuckit, M., Tipp, J., Reich, T., Hesselbrock, V. M., \& Bucholz, K. K. (1995). The histories of withdrawal convulsions and delirium tremens in 1648 alcohol depedent subjects. Society for the Study of Addiction to Alcohol and Other Drugs, 1336-1347. https://doi.org/0965-2140/95/101335-13

Sessler, C. N., Gosnell, M. S., Grap, M. J., Brophy, G. M., O’Neal, P. V., Keane, K. A., ... Elswick, R. K. (2002). The Richmond agitation-sedation scale. American Journal of Respiratory Critical Care Medicine, 166, 1338-1344. https://doi.org/10.1164/rccm.2107138

Sutton, L. J., \& Jutel, A. (2016). Alcohol withdrawal syndrome in critically ill patients: identification, assessment, and management [Entire issue]. Critical Care Nurse, 36(1). Retrieved from www.ccnonline.org

Trevisan, L. A., Boutros, N., Petrakis, I. L., \& Krystal, J. H. (1998). Complications of alcohol withdrawal. Alcohol Health and Research World, 22(1), 61-65. Retrieved from

Ungarian, J., Rankin, J., \& Then, K. (2019, August 4). Delirium in the Intensive Care Unit: Is Dexmedetomidine Effective? Critical Care Nurse, 39(4). https://doi.org/10.4037/ccn2019591

VanderWeide, L. A., Foster, C. J., MacLaren, R., Kiser, T. H., Fish, D. N., \& Mueller, S. W. (2016). Evaluation of early dexmedetomidine addition to the standard of care for severe alcohol wthdrawal in the ICU: a retrospective controlled cohort study. 
Journal of Intensive Care Medicine, 31, 198-204.

https://doi.org/10.1177/0885066614554908 
